\title{
Preventing numerical oscillations in the flux-split based finite difference method for compressible flows with discontinuities
}

\author{
Zhiwei He ${ }^{a}$, Yousheng Zhang ${ }^{a}$, Xinliang $\mathrm{Li}^{\mathrm{b}}$, $\mathrm{Li} \mathrm{Li}^{\mathrm{b}}$, Baolin Tian ${ }^{\mathrm{a}, *}$ \\ a Laboratory of Computational Physics, Institute of Applied Physics and Computational Mathematics, Beijing 100094, China \\ b State Key Laboratory of High-temperature Gas Dynamics, Institute of Mechanics, Chinese Academy of Sciences, Beijing 100190, China
}

\section{A R T I C L E I N F O}

\section{Article history:}

Received 4 January 2015

Received in revised form 3 July 2015

Accepted 25 July 2015

Available online 30 July 2015

\section{Keywords:}

Compressible flows

Contact discontinuity

Material interface

Numerical oscillations

Finite difference method

Flux vector splitting

WENO

\begin{abstract}
A B S T R A C T
In simulating compressible flows with contact discontinuities or material interfaces, numerical pressure and velocity oscillations can be induced by point-wise flux vector splitting (FVS) or component-wise nonlinear difference discretization of convection terms. The current analysis showed that the oscillations are due to the incompatibility of the point-wise splitting of eigenvalues in FVS and the inconsistency of component-wise nonlinear difference discretization among equations of mass, momentum, energy, and even fluid composition for multi-material flows. Two practical principles are proposed to prevent these oscillations: (i) convective fluxes must be split by a global FVS, such as the global Lax-Friedrichs FVS, and (ii) consistent discretization between different equations must be guaranteed. The latter, however, is not compatible with component-wise nonlinear difference discretization. Therefore, a consistent discretization method that uses only one set of common weights is proposed for nonlinear weighted essentially non-oscillatory (WENO) schemes. One possible procedure to determine the common weights is presented that provided good results. The analysis and methods stated above are appropriate for both single- (e.g., contact discontinuity) and multi-material (e.g., material interface) discontinuities. For the latter, however, the additional fluid composition equation should be split and discretized consistently for compatibility with the other equations. Numerical tests including several contact discontinuities and multi-material flows confirmed the effectiveness, robustness, and low computation cost of the proposed method.
\end{abstract}

(c) 2015 Elsevier Inc. All rights reserved.

\section{Introduction}

Flows evolving from contact discontinuities or material interfaces are present in a wide range of problems, such as inertial confinement fusion, supernova explosions, high-speed combustion, cavitation bubble clouds, industrial coatings, and fluidized beds. The Richtmyer-Meshkov instability [1] and Rayleigh-Taylor instability [2] are two archetypical examples. Such flows give rise to challenging problems both theoretically and computationally [3].

Much of the theoretical and practical evidence accumulated over many years suggest that, in the presence of shocks, there is no alternative but to use conservative methods [4]. However, anomalies or computational difficulties with using conservative methods have been reported, such as the low Mach number flow [5-10], sonic point glitch [11], carbuncle phenomenon [12], and others [4,13-16]. In this paper, we focus on the problems of compressible flows with contact discon-

\footnotetext{
* Corresponding author.

E-mail address: tian_baolin@iapcm.ac.cn (B. Tian).
} 
tinuities and/or material interfaces. Anomalies are also encountered when these problems are solved by using conservative methods.

First, numerical oscillations can be observed in problems with single-material contact discontinuities. As discussed by Clerc [17], when a conservative Godunov-type finite volume method (FVM) is used for these problems, spurious oscillations tend to be generated. These oscillations slow down the time-marching procedures for steady-state computations and spoil the numerical flow fields. Clerc analyzed the source of these oscillations and found that these oscillations are produced provided that the isobars are not a straight line. Johnsen [18] found that another kind of velocity and pressure oscillations is induced when high-order component-wise weighted essentially non-oscillatory (WENO) reconstruction is used. These oscillations are caused by the inconsistent reconstruction between the mass, momentum, and energy equations. The reconstruction of primitive or characteristic variables must be adopted [18].

Second, some computational difficulties are encountered when solving multi-material flows consisting of pure fluids separated by material interfaces. In these problems, an additional equation quantifying the fluid composition must be introduced to close the governing equations. In early algorithms for computing compressible multi-material flows, the discontinuous nature of the fluid composition was represented by the mass fraction, ratio of specific heats, or level-set function and evolved according to an advection equation coupled to the Euler equations [19]. However, Toro [4] found that, when the fluid composition is represented by the ratio of specific heats, pressure oscillations are introduced even by using various classical first-order schemes. Abgrall and Karni [3] found that, no matter what is used to represent the fluid composition, velocity and pressure oscillations already appear in the first-order computations and are not removed by going to a higher order. Any Godunov-type scheme that is fully conservative cannot maintain pressure equilibrium and will develop a pressure oscillation across material interfaces [20].

In order to overcome these difficulties, some fully nonconservative [21] and non-strictly conservative [20,22-25] approaches have been proposed. Among these attempts, the quasi-conservative approach proposed by Abgrall [20] seems promising and has been extended to problems with complicated equations of state [26-28]. The main idea of this approach is that the proper variable to represent the fluid composition is a function of the ratio of specific heats (i.e., $\Gamma=\frac{1}{\gamma-1}$, where $\gamma$ is the ratio of specific heats) and the equation of $\Gamma$ cannot be discretized independently of the discretization of the conserved variables in the Euler equations. This approach has been realized with the low-order finite volume method (FVM), such as first- and second-order variable reconstruction with various Riemann solvers [29]. Recently, this approach was extended to high-order WENO reconstruction with the HLLC solver [30] by Johnsen et al. [19,31]. In contrast to the low-order schemes, they found that new numerical oscillations are produced when high-order nonlinear WENO schemes are used. They analyzed the numerical oscillations and suggested that only the reconstruction of primitive variables can eliminate the spurious oscillations because the specific heat ratio is not constant [31]. Nonomura et al. [32] more comprehensively discussed the numerical oscillations of compressible multi-material flows and concluded that primitive variable interpolation can effectively eliminate numerical oscillations and that a better choice of fully conservative or quasi-conservative forms depends on the problem. Here, we emphasize that Nonomura et al. selected the weighted-compact-nonlinear-scheme (WCNS) variable interpolation finite difference formulation [33] to take over the numerical technique developed with the FVM [19].

On the other hand, for most Richtmyer-Meshkov instability and Rayleigh-Taylor instability problems, the initial configurations are unsteady, and the flows eventually evolve to turbulence. This remains a challenge for theoretical and experimental studies [34]. Instead, numerical simulations have become a powerful tool for these studies, particularly the direct numerical simulation of compressible turbulence. This requires the numerical methods to be high-fidelity in terms of accuracy, resolution, and capturing discontinuities. As a consequence, various high-order methods have been developed. Among these methods, the finite difference method (FDM), especially the high-order component-wise FDM [35], has been welcomed for its simplicity, effectiveness, and low computational cost [36-38].

However, in contrast to the frequent use of the FVM to simulate multi-material flows, the high-order FDM is used more often to simulate single-material flows. So far, there are just a few results computed with the FDM for compressible flows with contact discontinuities and/or material-interfaces. In 2003, Marquina and Mulet [39] directly solved the conservation form of the governing equations with the FDM. In this simulation, the convective fluxes were first split with Marquina's flux splitting method [40], and WENO schemes [41] were then used to obtain the numerical fluxes. Unfortunately, they observed spurious pressure and velocity oscillations at the material interfaces that were assumed to be too small to interfere with the physics of their simulation. Recently, Terashima et al. [42] directly implemented central finite difference schemes to simulate multi-material flows by introducing consistent local artificial diffusion terms to suppress the numerical oscillations of pressure and velocity.

To our knowledge, there has been no general analysis on the computational difficulties of the flux-split based FDM, especially for the frequently used nonlinear finite difference WENO schemes, when solving problems of compressible flows with contact discontinuities and/or material interfaces. As argued by Nonomura et al. [32], the technique proposed by Johnsen and Colonius [19] cannot be applied to the finite difference WENO schemes because these schemes do not include primitive variable reconstruction. In this paper, we present a systematic analysis of this question. The logic and main results are as follows:

- For compressible flows with contact discontinuities or material interfaces, nonphysical oscillations of the pressure and velocity will be produced as long as the implementation of the FDM involves either one of the following operations: (i) point-wise flux vector splitting (FVS) is used to split convective fluxes or (ii) a nonlinear finite difference scheme 
is used to construct numerical fluxes component by component. Under the framework of the FDM, additional constraint conditions to keep the oscillation-free property of pressure and velocity were derived based on Abgrall's physical principle [20]. The constraint conditions were used to analyze sources of oscillations, and two sources were found.

- The first source comes from point-wise FVS. Because of the intrinsic change in the speed of sound at discontinuities, the point-wise splitting of eigenvalues among the equations of mass, momentum, energy, and even fluid composition for multi-material flows is incompatible with the constraint conditions. When a global FVS (e.g., the global Lax-Friedrichs FVS) is used, however, the additional constraint conditions can be met automatically and thus prevent this type of oscillation.

- The second source comes from component-wise nonlinear difference discretization. When a nonlinear finite difference scheme (e.g., the WENO scheme) is used to discretize convection terms, the inconsistency of the component-wise discretization among the equations of mass, momentum, energy, and even fluid composition for multi-material flows is not compatible with the additional constraint conditions, and a consistent discretization must be guaranteed. A consistent implementation for WENO schemes is proposed that uses only one set of common weights to successfully prevent this type of oscillation.

- The numerical oscillations and methods stated above are appropriate for either single- (e.g., contact discontinuity) or multi-material (e.g., material interface) discontinuities. For the latter, however, the additional fluid composition equation should be split compatibly and discretized consistently with other equations. Numerical tests that included several contact discontinuities and multi-material flows confirmed the effectiveness, robustness, and low computation cost of our proposed method.

The paper is organized as follows. The governing equations and numerical methodology are given in Section 2, and the numerical oscillation analysis is presented in Section 3. The numerical tests and discussions are presented in Section 4, and the conclusions are given in Section 5.

\section{Governing equations and numerical methodology}

\subsection{Governing equations}

The governing equations are the compressible Euler equations:

$$
\begin{aligned}
& \frac{\partial \rho}{\partial t}+\frac{\partial \rho u}{\partial x}=0, \\
& \frac{\partial \rho u}{\partial t}+\frac{\partial\left(\rho u^{2}+p\right)}{\partial x}=0, \\
& \frac{\partial \rho E}{\partial t}+\frac{\partial(\rho E u+p u)}{\partial x}=0,
\end{aligned}
$$

where $\rho$ is the density, $u$ is the velocity, $p$ is the pressure, $E\left(=e+u^{2} / 2\right)$ is the total energy, and $e$ is the internal energy. To close the system, an equation of state such as the ideal gas law, i.e., $p=(\gamma-1) \rho e$, is adopted, where $\gamma$ is the ratio of specific heats. For compressible multi-material flows modeled in the Eulerian framework, additional equation(s) that quantify the evolution of the fluid composition should be added to close the system. Following Abgrall and Karni's analysis [3], the additional equation should be written as follows:

$$
\frac{\partial \Gamma}{\partial t}+u \frac{\partial \Gamma}{\partial x}=0
$$

\subsection{Numerical methodology}

In this work, component-wise finite difference discretization [35] was used. Eqs. (1)-(3) can be rewritten as

$$
\frac{\partial \mathbf{u}}{\partial t}+\frac{\partial \mathbf{f}}{\partial x}=0
$$

where $\mathbf{u}=(\rho, \rho u, \rho E)^{T}$ and $\mathbf{f}=\left(\rho u, \rho u^{2}+p, \rho E u+p u\right)^{T}$. Discretizing Eq. (5) with the FDM yields the following semidiscrete form:

$$
\frac{d \mathbf{u}_{i}}{d t}=-\frac{\widehat{\mathbf{f}}_{i+1 / 2}-\widehat{\mathbf{f}}_{i-1 / 2}}{\Delta x},
$$

where $\mathbf{u}_{i}$ is the point value at $i$ of an $N$-point grid with uniform grid spacing $\Delta x$ and $\widehat{\mathbf{f}}_{i \pm 1 / 2}$ is the numerical flux vectors.

The component-wise FDM is implemented in two steps [35]. The first step is to split the flux vector into the positive flux vector $\mathbf{f}^{+}$and negative flux vector $\mathbf{f}^{-}$as follows:

$$
\mathbf{f}(\mathbf{u})=\mathbf{f}^{+}(\mathbf{u})+\mathbf{f}^{-}(\mathbf{u})
$$


where the two Jacobians $\frac{\partial \mathbf{f}^{+}}{\partial \mathbf{u}}, \frac{\partial \mathbf{f}^{-}}{\partial \mathbf{u}}$ can still be diagonalized and have only non-negative/non-positive eigenvalues [35]. The second step is to use a finite difference scheme to construct the numerical fluxes $\widehat{\mathbf{f}}_{i \pm 1 / 2}$ component by component.

In this paper, we express the unified form of Steger-Warming FVS [43] and Lax-Friedrichs type FVS [44] as follows:

$$
\mathbf{f}^{ \pm}(\mathbf{u})=R \frac{\Lambda \pm \Lambda^{*}}{2} L \mathbf{u},
$$

where $R$ and $L$ are matrices formed by the right and left eigenvectors, respectively, of the Jacobian $\frac{\partial \mathbf{f}}{\partial \mathbf{u}}$ and $\Lambda$ is a diagonal matrix formed by the corresponding eigenvalues $\lambda_{j}(j=1, \cdots, 3)$ of $\frac{\partial \mathbf{f}}{\partial \mathbf{u}}$. In this paper, they are ordered as $\lambda_{1}=u-c, \lambda_{2}=u$, $\lambda_{3}=u+c$, where $c$ is the local speed of sound. The diagonal matrix $\Lambda^{*}$ formed by $\lambda_{j}^{*}(j=1, \cdots, 3)$ differs for the different FVS methods: (i) in the Steger-Warming FVS, $\Lambda^{*}=|\Lambda|$; (ii) in the Lax-Friedrichs type FVS, $\Lambda^{*}$ is a proper matrix [35].

\section{Numerical oscillation analysis}

\subsection{General principles for numerical oscillations analysis}

We adopted the principle introduced by Abgrall [20] to analyze the numerical oscillations. The main idea of this principle can be summarized as follows: for compressible flows with contact discontinuities [18] or material interfaces [20], if the velocity and pressure at the time level $t^{n}$ share $p_{i}^{n}=p, u_{i}^{n}=u(i=1, \ldots, N)$, they should stay constant at the next time level $t^{n+1}$. That is,

$$
\begin{aligned}
& u_{i}^{n+1}=u_{i}^{n}=u, \\
& p_{i}^{n+1}=p_{i}^{n}=p .
\end{aligned}
$$
give

Note that $u_{i}^{n+1}$ and $p_{i}^{n+1}$ are updated by integrating Eq. (6), which can be integrated with the explicit Euler scheme to

$$
\mathbf{u}_{i}^{n+1}=\mathbf{u}_{i}^{n}-\frac{\Delta t}{2 \Delta x} \mathbf{F}_{i},
$$

where $\mathbf{F}_{i}=\left(F_{i}^{\rho}, F_{i}^{\rho u}, F_{i}^{\rho E}\right)^{T}$ is defined as

$$
\mathbf{F}_{i}=2\left(\widehat{\mathbf{f}}_{i+1 / 2}-\widehat{\mathbf{f}}_{i-1 / 2}\right) \text {, }
$$

and the coefficient $\frac{1}{2}$ is extracted to simplify the following derivation. By expanding Eq. (11) with definitions, we can obtain

$$
\begin{aligned}
u_{i}^{n+1} & =\frac{(\rho u)_{i}^{n+1}}{\rho_{i}^{n+1}}=\frac{(\rho u)_{i}^{n}-F_{i}^{\rho u} \Delta t /(2 \Delta x)}{\rho_{i}^{n}-F_{i}^{\rho} \Delta t /(2 \Delta x)}, \\
\left(\frac{p}{\gamma-1}\right)_{i}^{n+1} & =(\rho E)_{i}^{n+1}-\frac{\left[(\rho u)_{i}^{n+1}\right]^{2}}{2 \rho_{i}^{n+1}} \\
& =(\rho E)_{i}^{n}-F_{i}^{\rho E} \frac{\Delta t}{2 \Delta x}-\frac{\left[(\rho u)_{i}^{n}-F_{i}^{\rho u} \Delta t /(2 \Delta x)\right]^{2}}{2\left[\rho_{i}^{n}-F_{i}^{\rho} \Delta t /(2 \Delta x)\right]} .
\end{aligned}
$$

This will frequently be used in this paper to derive the additional constraint conditions required for the expected oscillationfree property.

\subsection{Numerical oscillations source I: flux vector splitting}

\subsubsection{Numerical oscillations induced by point-wise flux vector splitting}

In this subsection, we use the simplest example of a problem to demonstrate the numerical oscillations induced by FVS: a stationary contact discontinuity discretized by the first-order upwind difference scheme.

Under the condition that the first-order upwind finite difference scheme is used, the flux-split based positive and negative numerical fluxes are $\widehat{\mathbf{f}}_{i+1 / 2}^{+}=\mathbf{f}_{i}^{+}$and $\widehat{\mathbf{f}}_{i+1 / 2}^{-}=\mathbf{f}_{i+1}^{-}$, respectively. Thus, we get

$$
\widehat{\mathbf{f}}_{i+1 / 2}=\mathbf{f}_{i}^{+}+\mathbf{f}_{i+1}^{-} \text {. }
$$

A more elegant approach is to follow Harten et al.'s method [45] to define

$$
\mathbf{f}^{\varepsilon}(\mathbf{u})=\mathbf{f}^{+}(\mathbf{u})-\mathbf{f}^{-}(\mathbf{u}) .
$$

If we refer to Eq. (8), we get $\mathbf{f}^{\varepsilon}=R \Lambda^{*} L \mathbf{u}$. Combining Eqs. (16) and (7) give 


$$
\begin{aligned}
& \mathbf{f}^{+}(\mathbf{u})=\left(\mathbf{f}(\mathbf{u})+\mathbf{f}^{\epsilon}(\mathbf{u})\right) / 2, \\
& \mathbf{f}^{-}(\mathbf{u})=\left(\mathbf{f}(\mathbf{u})-\mathbf{f}^{\epsilon}(\mathbf{u})\right) / 2 .
\end{aligned}
$$

Substituting the two relations into Eq. (15) gives

$$
\widehat{\mathbf{f}}_{i+1 / 2}=\frac{1}{2}\left(\left(\mathbf{f}_{i+1}+\mathbf{f}_{i}\right)-\left(\mathbf{f}_{i+1}^{\varepsilon}-\mathbf{f}_{i}^{\varepsilon}\right)\right) .
$$

For the problem considered in this subsection of a stationary contact discontinuity, we get

$$
\begin{aligned}
& \gamma=\text { const } \\
& u=0 .
\end{aligned}
$$

By expanding the right-hand side of Eq. (19) with Eqs. (20) and (21), we can obtain

$$
\widehat{\mathbf{f}}_{i+1 / 2}=\left(\begin{array}{c}
0 \\
p \\
0
\end{array}\right)-\frac{1}{2}\left(\begin{array}{c}
p\left(\left(\frac{\lambda_{1}^{*}+\lambda_{3}^{*}}{2 c^{2}}\right)_{i+1}-\left(\frac{\lambda_{1}^{*}+\lambda_{3}^{*}}{2 c^{2}}\right)_{i}\right)+S_{i+1 / 2} \\
p\left(\left(\frac{\lambda_{3}^{*}-\lambda_{1}^{*}}{2 c}\right)_{i+1}-\left(\frac{\lambda_{3}^{*}-\lambda_{1}^{*}}{2 c}\right)_{i}\right) \\
p\left(\left(\frac{\lambda_{3}^{*}+\lambda_{1}^{*}}{2(\gamma-1)}\right)_{i+1}-\left(\frac{\lambda_{3}^{*}+\lambda_{1}^{*}}{2(\gamma-1)}\right)_{i}\right)
\end{array}\right)
$$

where $S_{i+1 / 2}=\left(\lambda_{2}^{*}\left(\rho-\frac{p}{c^{2}}\right)\right)_{i+1}-\left(\lambda_{2}^{*}\left(\rho-\frac{p}{c^{2}}\right)\right)_{i}$. For this problem, the sufficient and necessary conditions to prevent the nonphysical oscillations of the velocity and pressure are $F_{i}^{\rho u}=0$ and $F_{i}^{\rho E}=0$. By using Eqs. (22) and (12), we can obtain

$$
\begin{aligned}
& {\left[\left(\frac{\left(\lambda_{3}^{*}-\lambda_{1}^{*}\right)}{2 c}\right)_{i+1}-\left(\frac{\left(\lambda_{3}^{*}-\lambda_{1}^{*}\right)}{2 c}\right)_{i}\right]-\left[\left(\frac{\left(\lambda_{3}^{*}-\lambda_{1}^{*}\right)}{2 c}\right)_{i}-\left(\frac{\left(\lambda_{3}^{*}-\lambda_{1}^{*}\right)}{2 c}\right)_{i-1}\right]=0,} \\
& {\left[\left(\lambda_{1}^{*}+\lambda_{3}^{*}\right)_{i+1}-\left(\lambda_{1}^{*}+\lambda_{3}^{*}\right)_{i}\right]-\left[\left(\lambda_{1}^{*}+\lambda_{3}^{*}\right)_{i}-\left(\lambda_{1}^{*}+\lambda_{3}^{*}\right)_{i-1}\right]=0 .}
\end{aligned}
$$

Based on the constraint conditions of Eqs. (23) and (24), we can conclude the following:

- The general conditions to prevent the nonphysical oscillations of velocity and pressure in this problem is that $\left(\lambda_{1}^{*}\right)_{i}=$ $\left(\lambda_{3}^{*}\right)_{i}\left(\equiv\left(\lambda_{0}^{*}\right)_{i}\right)$ for the fixed point $x_{i}$ and $\left(\lambda_{0}^{*}\right)_{m}$ should stay constant for an arbitrary $m$, where $m(=i-l, \cdots, i+r)$ labels the points of the stencil of the scheme from left to right.

- In the FVM, the state on either side of each cell edge is reconstructed by using cell averages to yield a Riemann problem with left and right states. An exact/approximate Riemann solver or FVS method such as the Steger-Warming flux [46], Lax-Friedrichs flux or Rusanov flux [47] is then used to provide the upwind numerical flux [47]. However, the high-order flux-split based FDM needs a FVS method to split the convective flux pointwise in the first step. For pointwise FVS, $\left(\lambda_{j}^{*}\right)_{i}(j=1, \cdots, 3 ; i=1, \cdots, N)$ is usually different for either different equations (i.e., $\left.\left(\lambda_{1}^{*}\right)_{i} \neq\left(\lambda_{2}^{*}\right)_{i} \neq\left(\lambda_{3}^{*}\right)_{i}\right)$ or different grid points (e.g., for this problem, $\left(\lambda_{j}^{*}\right)_{i-1} \neq\left(\lambda_{j}^{*}\right)_{i} \neq\left(\lambda_{j}^{*}\right)_{i+1}$ near the discontinuity). Thus, it is impossible to meet the constraint conditions (Eqs. (23) and (24)) at each time step, and numerical oscillations are generated. For example, in the Steger-Warming FVS, $\lambda_{1}^{*}=\lambda_{3}^{*}=c, \lambda_{2}^{*}=0$, and pressure oscillations are first generated at the location of the discontinuity where $c_{i+1}-2 c_{i}+c_{i-1} \neq 0$ (see Eq. (24)) to spoil the velocity at the next time step.

- With proper choice of $\Lambda^{*}$, the Lax-Friedrichs type FVS methods proposed by Shu [35,44] can satisfy the general conditions. Among the Lax-Friedrichs type FVS methods, the global Lax-Friedrichs FVS is the most simple and efficient method to satisfy these constraints. Here, $\Lambda^{*}=\alpha I$, where $I$ is the identity matrix and $\alpha=\max _{1 \leq j \leq 3}\left|\lambda_{j}\right|$ over the whole computation domain [35]. It should be noted that the global Lax-Friedrichs FVS is the most diffusive. For lower order methods, there is a big difference between results obtained by different FVS methods. However, this difference becomes smaller for high order finite difference methods [35]. In the following sections, the global Lax-Friedrichs FVS is used as the foundation for other analyses.

\subsubsection{Prevent numerical oscillations with global flux vector splitting}

In the previous subsection, we showed that the oscillations are essentially generated with point-wise FVS. In this subsection, we show that the global Lax-Friedrichs FVS coupled with arbitrary linear difference schemes can successfully suppress the oscillations for a general problem of stationary/nonstationary contact discontinuities or material interfaces.

For general linear finite difference schemes, the positive and negative numerical fluxes $\widehat{\mathbf{f}}_{i+1 / 2}^{ \pm}$can be written with finite difference operators $D_{i+1 / 2}^{ \pm}$as $\widehat{\mathbf{f}}_{i+1 / 2}^{ \pm}=D_{i+1 / 2}^{ \pm}\left(\mathbf{f}^{ \pm}\right)$. Thus, we get

$$
\widehat{\mathbf{f}}_{i+1 / 2}=D_{i+1 / 2}^{+}\left(\mathbf{f}^{+}\right)+D_{i+1 / 2}^{-}\left(\mathbf{f}^{-}\right) .
$$


For example, the $(2 r-1)$-order linear upwind finite difference scheme can be written as

$$
\widehat{\mathbf{f}}_{i+1 / 2}=\sum_{l=-(r-1)}^{r-1} a_{l} \mathbf{f}_{i+l}^{+}+\sum_{l=-(r-2)}^{r} b_{l} \mathbf{f}_{i+l}^{-},
$$

where $a_{l}$ and $b_{l}$ are constant coefficients.

With the global Lax-Friedrichs FVS and the conditions of $u_{i}^{n}=u$ and $p_{i}^{n}=p(i=1, \ldots, N)$, substituting Eq. (25) into Eq. (12) yields

$$
\begin{aligned}
F_{i}^{\rho}= & D_{i+1 / 2}^{+}(\rho u+\rho \alpha)+D_{i+1 / 2}^{-}(\rho u-\rho \alpha) \\
& -D_{i-1 / 2}^{+}(\rho u+\rho \alpha)-D_{i-1 / 2}^{-}(\rho u-\rho \alpha), \\
F_{i}^{\rho u}= & u F_{i}^{\rho}, \\
F_{i}^{\rho E}= & p Q_{i}+\frac{u^{2}}{2} F_{i}^{\rho},
\end{aligned}
$$

where

$$
\begin{aligned}
Q_{i}= & D_{i+1 / 2}^{+}\left(\frac{u+\alpha}{\gamma-1}\right)+D_{i+1 / 2}^{-}\left(\frac{u-\alpha}{\gamma-1}\right) \\
& -D_{i-1 / 2}^{+}\left(\frac{u+\alpha}{\gamma-1}\right)-D_{i-1 / 2}^{-}\left(\frac{u-\alpha}{\gamma-1}\right) .
\end{aligned}
$$

By substituting Eq. (28) into Eq. (13), we can obtain

$$
u_{i}^{n+1}=u_{i}^{n}=u .
$$

Obviously, Eq. (31) is consistent with Eq. (9), and the oscillation-free property for the velocity is guaranteed.

By substituting Eq. (29) into Eq. (14) with Eq. (31), we can obtain

$$
\begin{aligned}
\left(\frac{p}{\gamma-1}\right)_{i}^{n+1}= & \left(\frac{p}{\gamma-1}\right)_{i}^{n}-\frac{\Delta t}{\Delta x} \frac{u+\alpha}{2}\left(D_{i+1 / 2}^{+}\left(\frac{p}{\gamma-1}\right)-D_{i-1 / 2}^{+}\left(\frac{p}{\gamma-1}\right)\right) \\
& -\frac{\Delta t}{\Delta x} \frac{u-\alpha}{2}\left(D_{i+1 / 2}^{-}\left(\frac{p}{\gamma-1}\right)-D_{i-1 / 2}^{-}\left(\frac{p}{\gamma-1}\right)\right) .
\end{aligned}
$$

For single-material flows (i.e., $\gamma=$ const and $\left.D_{i \pm 1 / 2}^{ \pm}\left(\frac{p}{\gamma-1}\right)=\frac{p}{\gamma-1}\right)$, Eq. (32) gives $p_{i}^{n+1}=p_{i}^{n}=p$. This meets the constraint condition of Eq. (10). For multi-material flows (i.e., $\gamma \neq$ const), to meet the expected constraint condition of $p_{i}^{n+1}=p_{i}^{n}=p$ for an arbitrary pressure, Eq. (32) implies that the following discretized relation should be strictly guaranteed:

$$
\begin{aligned}
\left(\frac{1}{\gamma-1}\right)_{i}^{n+1}= & \left(\frac{1}{\gamma-1}\right)_{i}^{n}-\frac{\Delta t}{\Delta x} \frac{u+\alpha}{2}\left(D_{i+1 / 2}^{+}\left(\frac{1}{\gamma-1}\right)-D_{i-1 / 2}^{+}\left(\frac{1}{\gamma-1}\right)\right) \\
& -\frac{\Delta t}{\Delta x} \frac{u-\alpha}{2}\left(D_{i+1 / 2}^{-}\left(\frac{1}{\gamma-1}\right)-D_{i-1 / 2}^{-}\left(\frac{1}{\gamma-1}\right)\right) .
\end{aligned}
$$

This is just the discrete form of the following equation:

$$
\frac{\partial \Gamma}{\partial t}+\frac{u+\alpha}{2}\left(\frac{\partial \Gamma}{\partial x}\right)^{+}+\frac{u-\alpha}{2}\left(\frac{\partial \Gamma}{\partial x}\right)^{-}=0 .
$$

In other words, to prevent the numerical oscillation of compressible multi-material flows with material interfaces, the fluid composition equation should be solved with the global Lax-Friedrichs FVS as Eq. (34).

We can conclude the following:

- The specific expressions of $D_{i \pm 1 / 2}^{ \pm}$are not needed in the analysis above. Therefore, for an arbitrary linear finite difference schemes, the use of global Lax-Friedrichs FVS can indeed avoid the oscillations induced by point-wise FVS.

- For the analysis presented above, one condition is already used implicitly: $D_{i \pm 1 / 2}^{ \pm}$takes a consistent expression for the discretization of the equations of mass, momentum, energy, and even fluid composition for multi-component flows. This condition only works for linear finite difference schemes, not for nonlinear finite difference schemes. For the latter, oscillations will be induced. See the analysis presented in the next section. 


\subsection{Numerical oscillations source II: component-wise nonlinear difference discretization}

As pointed out previously, there are two steps in the component-wise FDM. In Section 3.2, we analyzed the oscillations induced by the first step. In this section, we show that oscillations are also induced by the use of nonlinear difference schemes. Simple calculations can be used to verify that the constraint conditions are not satisfied if the linear difference operator introduced in Section 3.2 is replaced by a nonlinear difference operator. In the following subsection, we show that numerical oscillations can come from the inconsistency of component-wise nonlinear discretization among the equations of mass, momentum, energy, and even fluid composition for multi-material flows.

\subsubsection{Numerical oscillations induced by nonlinear WENO scheme}

We first introduce the rules adopted in this paper: the weights of the $(2 r-1)$-order WENO scheme [41,48] are denoted by $\omega_{l, i+1 / 2, k}^{ \pm}$, where $l$ ranging from 1 to $r$ represents the lth sub-stencil of the WENO scheme, $k$ ranging from 1 to 3 (or 4 ) represents the equations of mass, momentum, energy (and even fluid composition for multi-material flows), respectively, and the subscript \pm represents the positive or negative fluxes obtained by the global Lax-Friedrichs FVS method. The smooth indicators of the WENO scheme are denoted as $I S_{l, i+1 / 2, k}^{ \pm}$, and the finite difference operator of the $r$ th sub-scheme is denoted as $D_{l, i+1 / 2}^{ \pm}$.

Similar to Section 3.2.1, we first derive the specific expression of $\mathbf{F}_{i}$ in Eq. (11). With the global Lax-Friedrichs FVS and the conditions $u_{i}^{n}=u$ and $p_{i}^{n}=p(i=1, \ldots, N)$, the following can be obtained:

$$
\begin{aligned}
F_{i}^{\rho} & =\sum_{l=1}^{r} \omega_{l, i+1 / 2,1}^{+} D_{l, i+1 / 2}^{+}(\rho u+\rho \alpha)+\sum_{l=1}^{r} \omega_{l, i+1 / 2,1}^{-} D_{l, i+1 / 2}^{-}(\rho u-\rho \alpha) \\
& -\sum_{l=1}^{r} \omega_{l, i-1 / 2,1}^{+} D_{l, i-1 / 2}^{+}(\rho u+\rho \alpha)-\sum_{l=1}^{r} \omega_{l, i-1 / 2,1}^{-} D_{l, i-1 / 2}^{-}(\rho u-\rho \alpha), \\
F_{i}^{\rho u} & =u M_{i, 1}+M_{i, 2}, \\
F_{i}^{\rho E} & =p S_{i, 1}+\frac{u^{2}}{2} S_{i, 2}+S_{i, 3},
\end{aligned}
$$

where

$$
\begin{aligned}
& M_{i, 1}=\sum_{l=1}^{r} \omega_{l, i+1 / 2,2}^{+} D_{l, i+1 / 2}^{+}(\rho u+\rho \alpha)+\sum_{l=1}^{r} \omega_{l, i+1 / 2,2}^{-} D_{l, i+1 / 2}^{-}(\rho u-\rho \alpha) \\
& -\sum_{l=1}^{r} \omega_{l, i-1 / 2,2}^{+} D_{l, i-1 / 2}^{+}(\rho u+\rho \alpha)-\sum_{l=1}^{r} \omega_{l, i-1 / 2,2}^{-} D_{l, i-1 / 2}^{-}(\rho u-\rho \alpha), \\
& M_{i, 2}=\sum_{l=1}^{r} \omega_{l, i+1 / 2,2}^{+} D_{l, i+1 / 2}^{+}(p)+\sum_{l=1}^{r} \omega_{l, i+1 / 2,2}^{-} D_{l, i+1 / 2}^{-}(p) \\
& -\sum_{l=1}^{r} \omega_{l, i-1 / 2,2}^{+} D_{l, i-1 / 2}^{+}(p)-\sum_{l=1}^{r} \omega_{l, i-1 / 2,2}^{-} D_{l, i-1 / 2}^{-}(p), \\
& S_{i, 1}=\sum_{l=1}^{r} \omega_{l, i+1 / 2,3}^{+} D_{l, i+1 / 2}^{+}\left(\frac{u+\alpha}{\gamma-1}\right)+\sum_{l=1}^{r} \omega_{l, i+1 / 2,3}^{-} D_{l, i+1 / 2}^{-}\left(\frac{u-\alpha}{\gamma-1}\right) \\
& -\sum_{l=1}^{r} \omega_{l, i-1 / 2,3}^{+} D_{l, i-1 / 2}^{+}\left(\frac{u+\alpha}{\gamma-1}\right)-\sum_{l=1}^{r} \omega_{l, i-1 / 2,3}^{-} D_{l, i-1 / 2}^{-}\left(\frac{u-\alpha}{\gamma-1}\right), \\
& S_{i, 2}=\sum_{l=1}^{r} \omega_{l, i+1 / 2,3}^{+} D_{l, i+1 / 2}^{+}(\rho u+\rho \alpha)+\sum_{l=1}^{r} \omega_{l, i+1 / 2,3}^{-} D_{l, i+1 / 2}^{-}(\rho u-\rho \alpha) \\
& -\sum_{l=1}^{r} \omega_{l, i-1 / 2,3}^{+} D_{l, i-1 / 2}^{+}(\rho u+\rho \alpha)-\sum_{l=1}^{r} \omega_{l, i-1 / 2,3}^{-} D_{l, i-1 / 2}^{-}(\rho u-\rho \alpha), \\
& S_{i, 3}=\sum_{l=1}^{r} \omega_{l, i+1 / 2,3}^{+} D_{l, i+1 / 2}^{+}(p u)+\sum_{l=1}^{r} \omega_{l, i+1 / 2,3}^{-} D_{l, i+1 / 2}^{-}(p u) \\
& -\sum_{l=1}^{r} \omega_{l, i-1 / 2,3}^{+} D_{l, i-1 / 2}^{+}(p u)-\sum_{l=1}^{r} \omega_{l, i-1 / 2,3}^{-} D_{l, i-1 / 2}^{-}(p u) .
\end{aligned}
$$


Obviously, the constraint conditions of Eqs. (9) and (10) cannot be met because the weights $\omega_{l, i+1 / 2, k}^{ \pm}(k=1,2,3)$ are usually different.

\subsubsection{Prevent numerical oscillations with consistent WENO implementation}

Although Eqs. (9) and (10) cannot be met for nonlinear WENO schemes, the expected relation of $u_{i}^{n+1}=u_{i}^{n}=u$ can be recovered if the following relation is guaranteed:

$$
\omega_{l, i+1 / 2,1}^{ \pm}=\omega_{l, i+1 / 2,2}^{ \pm} \text {. }
$$

This can be verified by substituting Eq. (36) to Eq. (13) and then to Eq. (9).

Similarly, under the condition of Eq. (43), the substitution of Eqs. (36) and (37) to Eq. (14) yields

$$
\left(\frac{p}{\gamma-1}\right)_{i}^{n+1}=\left(\frac{p}{\gamma-1}\right)_{i}^{n}-\frac{\Delta t}{2 \Delta x} p S_{i, 1} .
$$

Based on this, the additional requirements to meet the constraint conditions of Eq. (10) can be derived as follows:

- For single-material flows (i.e., $\gamma=$ const and $S_{i, 1}=0$ ), Eq. (44) shows that $p_{i}^{n+1}=p_{i}^{n}=p$ can be guaranteed if $\omega_{l, i+1 / 2,1}^{ \pm}=\omega_{l, i+1 / 2,3}^{ \pm}$. This can be combined with Eq. (43) to give $\omega_{l, i+1 / 2,1}^{ \pm}=\omega_{l, i+1 / 2,2}^{ \pm}=\omega_{l, i+1 / 2,3}^{ \pm}$. This means that only one set of common weights, denoted with $\omega_{l, i+1 / 2,0}^{ \pm}$, should be used. This is in contrast to the equation-numbermatched sets of weights in component-wise WENO discretization.

- For multi-material flows (i.e., $\gamma \neq$ const), similar to the derivation in Section 3.2 .2 (see Eqs. (33)-(34)), combining Eqs. (44) and (14) show that the expected relation of $p_{i}^{n+1}=p_{i}^{n}=p$ can be guaranteed if and only if the fluid composition is split with the global Lax-Friedrichs FVS as given in Eq. (34).

Therefore, in the traditional component-wise WENO implementation, the inconsistency of the WENO weights between the equations of mass, momentum, energy, and even fluid composition for multi-material flows may lead to spurious oscillations in the velocity and pressure. To prevent this kind of oscillation, a set of common weights $\omega_{l, i+1 / 2,0}^{ \pm}$should be used.

In terms of the theoretical analysis above, we can prevent the numerical oscillations as long as one set of common WENO weights is used in the WENO implementation. Thus, there are many possibilities for determining $\omega_{l, i+1 / 2,0}^{ \pm}$. However, this is not a trivial matter because the requirement of common weights $\omega_{l, i+1 / 2,0}^{ \pm}$is too strict for practical applications. Therefore, additional factors such as the stability and intrinsic nonlinear mechanism of WENO schemes must be considered when determining $\omega_{l, i+1 / 2,0}^{ \pm}$. Based on the results of our numerical tests, we suggest determining the final common weights $\omega_{l, i+1 / 2,0}^{ \pm}$as follows (without losing generality, we take the positive flux as an example):

(1) Define the smooth factor $\beta_{i+1 / 2, k}^{+}$of the positive flux as

$$
\beta_{i+1 / 2, k}^{+}=\frac{\sum_{l=1}^{r}\left(I S_{l, i+1 / 2, k}^{+}+\epsilon\right)}{\min \left(I S_{1, i+1 / 2, k}^{+}, \cdots, I S_{r, i+1 / 2, k}^{+}\right)+\epsilon},
$$

where $\epsilon$ is an adaptive number calculated by the primary $\epsilon$-adaptivity technique [49]. This is used to reduce the generation of spurious oscillations caused by the intrinsic mechanism of nonlinear WENO schemes (see the later discussion):

$$
\epsilon= \begin{cases}\epsilon_{\max } & \tau_{2 r-1} \leq S_{\min } \\ \epsilon_{\min } & \tau_{2 r-1} \geq S_{\max } \\ \frac{\epsilon_{\min }-\epsilon_{\max }}{S_{\max }-S_{\min }}\left(\tau_{2 r-1}-S_{\min }\right)+\epsilon_{\max } & \text { otherwise, }\end{cases}
$$

where $\epsilon_{\min }=10^{-6}, \epsilon_{\max }=10^{-2}, S_{\min }=10^{-3}$, and $S_{\max }=10^{-1} \cdot \tau_{2 r-1}$ is the $(2 r-1)$-order global smoothness indicators used in WENO-Z schemes [50-52].

(2) Calculate the $\beta_{i+1 / 2, k}^{+}$for equations of mass, energy equations, and even fluid composition for multi-material flows, but exclude the momentum equation. The momentum equation is excluded to consider the nonlinear stability [53,54] (see the later discussion).

(3) Select the equation with the maximal value of $\beta_{i+1 / 2, k}^{+}$(denoted with $k_{0}$ ), and then use the smooth indicators $I S_{l, i+1 / 2, k_{0}}^{+}$ of this equation to calculate the final common weights $\omega_{l, i+1 / 2,0}^{+}$. There are several methods to calculate the nonlinear weights $[41,50,55]$. In this paper, we use the formulations given in $[41,48]$ :

$$
\alpha_{l}^{+}=\frac{C_{l}^{+}}{\left(I S_{l, i+1 / 2, k_{0}}^{+}+\epsilon\right)^{2}}, \omega_{l, i+1 / 2,0}^{+}=\frac{\alpha_{l}^{+}}{\sum_{l=1}^{r} \alpha_{l}^{+}}, l=1, \cdots, r,
$$

where the coefficients $C_{l}^{+}$are optimal weights [41] and $\epsilon$ is set as shown in Eq. (46).

(4) Use the final common weights $\omega_{l, i+1 / 2,0}^{+}$to calculate the positive numerical flux $\widehat{\mathbf{f}}_{i+1 / 2}^{+}$. 
Here, we explain this consistency strategy for WENO schemes. First, the quantity $\beta_{i+1 / 2, k}^{+}$is defined to measure the smoothness of a local flow field. In the smooth region, the smooth factor $\beta_{i+1 / 2, k}^{+}$is $r$; in the region with a discontinuity, however, a stronger discontinuity means a larger $\beta_{i+1 / 2, k}^{+}$value. Therefore, to better capture the discontinuity, the set of WENO weights of the equation holding the strongest discontinuity should be used among the equations of mass, momentum, energy, and fluid composition for multi-material flows. This is the logic behind the introduction of $\beta_{i+1 / 2, k}^{+}$in Eq. (45). Second, if the initial velocity of an unsteady flow is zero, we will encounter the low Mach number problem [5,8-10]. It is well-known that the pressure gradient term in the momentum equation becomes singular as the Mach number approaches zero, and a remarkable roundoff error will be generated [53,54]. According to our numerical tests, this roundoff error results in a start-up instability. Therefore, the momentum equation is excluded, and this treatment greatly enhances the robustness of the current method while reducing the computation cost. Third, if a scheme is nonlinear, numerical oscillations may come from its intrinsic nonlinear mechanism. For the specific example of WENO schemes considered in this paper, even for a simple linear equation such as the linear advection equation, the nonlinearity of WENO schemes can generate spurious high modes oscillations. This was pointed out by Jia et al. [49] and validated in our numerical tests. These numerical oscillations will be generated regardless of the value of $\epsilon$, and the amplitude of the spurious oscillations increases with smaller $\epsilon$ values. The oscillations of these spurious high modes are inherent to nonlinear WENO schemes [49]. In order to reduce the generation of spurious oscillations caused by the intrinsic mechanism of nonlinear WENO schemes, a primary $\epsilon$-adaptivity technique has been suggested [49], and we adopted a simplified version of this technique, which is given in Eq. (46). By considering these factors, our consistent WENO implementation successfully prevents numerical oscillations. Numerical tests confirmed the robustness, effectiveness and low computation cost of the current method.

We can conclude the following:

- Independent of the FVS method used in the first step, nonphysical oscillations of the pressure and velocity can be observed if nonlinear difference discretization is implemented component by component. For nonlinear WENO schemes, our analysis showed that these numerical oscillations are caused by the inconsistent WENO weights used to calculate the convection terms for the equations of mass, momentum, energy, and fluid composition for multi-material flows.

- To prevent the numerical oscillations with WENO schemes, we suggest replacing the traditional component-wise WENO schemes with our consistent WENO implementation, where only one set of common WENO weights is used.

- All of the analysis presented above is implicitly based on the global Lax-Friedrichs FVS. Consequently, in order to successfully prevent the numerical oscillations of flux-split based component-wise finite difference discretization, the global Lax-Friedrichs FVS and consistent WENO implementation must be used simultaneously.

\section{Numerical tests and discussion}

Several tests were performed to test the proposed methods. In the rest of this paper, "SW" and "GLF" refer to Steger-Warming FVS and global Lax-Friedrichs FVS, respectively. "Current method" denotes the proposed combination of the GLF and consistent WENO schemes. For problems of multi-material flows, the additional fluid composition equation was solved in the nonconservative form (see Eq. (34)). The classical fifth-order WENO scheme [41] was used. A third-order TVD Runge-Kutta scheme [56] was used for time integration, and the CFL number was set to 0.6.

\subsection{Isolated contact discontinuity problem}

The first test was an isolated contact discontinuity problem. We used this test to reveal the numerical oscillations, confirm our previous analysis, and validate our proposed method. The initial conditions were

$$
(\rho, u, p, \gamma)= \begin{cases}(10,1,1 / \gamma, 1.4), & 0 \leq x \leq 0.1 \\ (1,1,1 / \gamma, 1.4), & 0.1<x \leq 2\end{cases}
$$

In order to eliminate the effects of boundaries and show the numerical oscillations more clearly in the various numerical implementations considered for this problem, the computation domain was set to [0,2], and a uniform mesh with 401 grid points (grid spacing: $\Delta x=0.005$ ) was used. The initial values were fixed at the left and right boundaries, and the results at $t=0.4$ are shown here and analyzed.

First, to exclude the possible numerical oscillations caused by nonlinear discretizations, linear difference schemes were used. Specifically, the fifth-order upwind linear difference scheme ( $r=3$ in Eq. (26)), which is denoted by "UD5", was used in the implementations of "SW+UD5" and "GLF+UD5". Figs. 1 and 2 show the profiles of the density, velocity, and pressure for the implementations of SW+UD5 and GLF+UD5. The profiles of the theoretical solutions are also plotted for comparison. According to our analysis, numerical oscillations in either the pressure or velocity would be expected with SW. This can be confirmed by Fig. 2, where distinct numerical oscillations can be observed near the location of the contact discontinuity $(x=0.5)$. In contrast, these nonphysical oscillations were successfully prevented when GLF was used. Therefore, Fig. 2 confirms that numerical oscillations are induced by the point-wise FVS method and that the use of GLF can successfully prevent this type of numerical oscillation. 


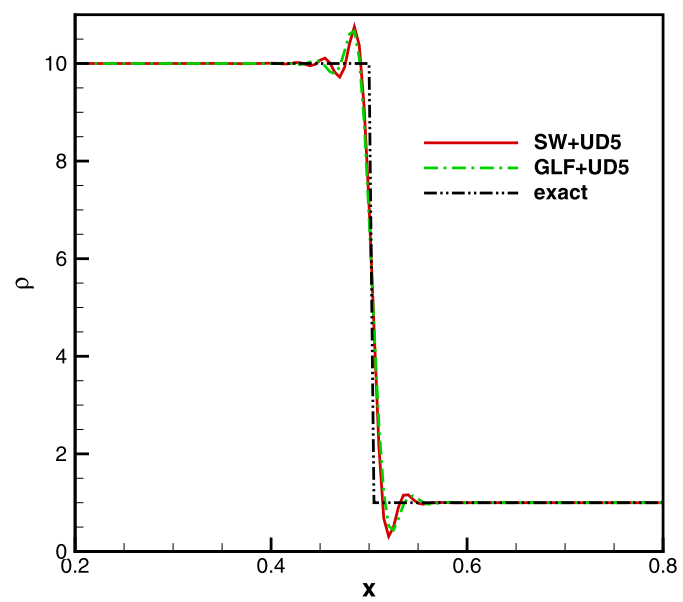

Fig. 1. Profiles of density at $t=0.4$ for implementations of "SW+UD5" and "GLF+UD5".
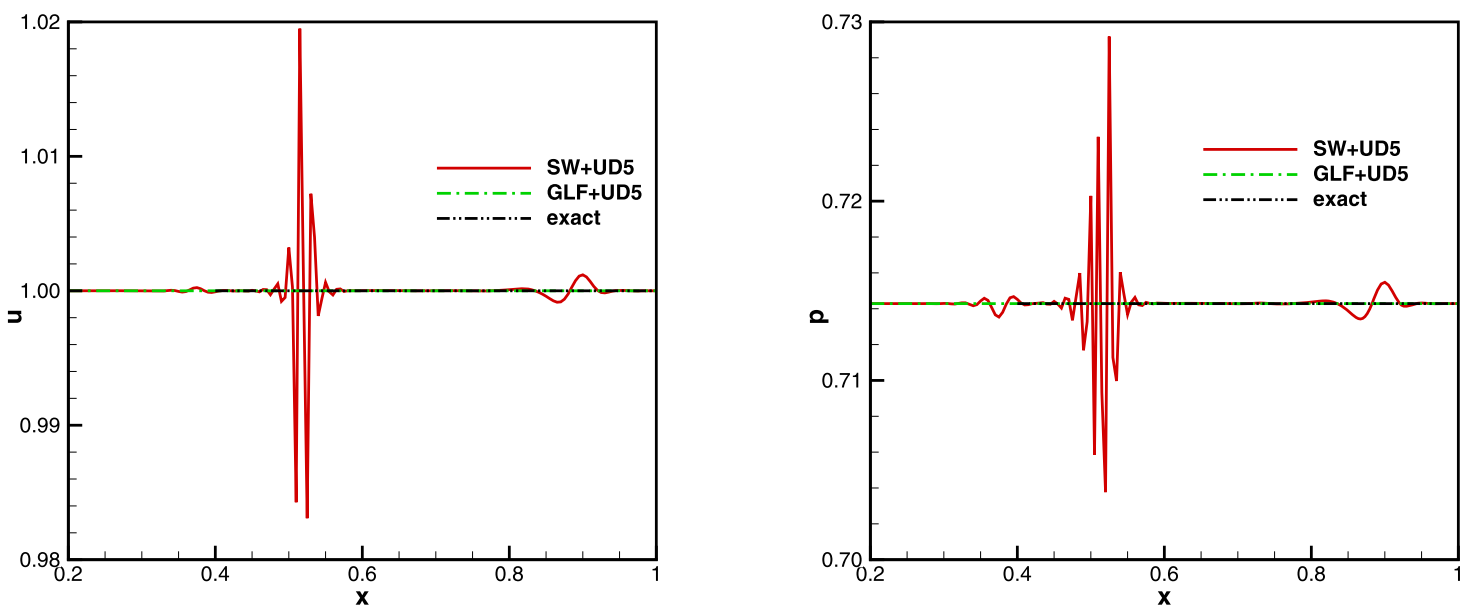

Fig. 2. Profiles of velocity (left) and pressure (right) at $t=0.4$ for implementations of "SW+UD5" and "GLF+UD5".

Figs. 3 and 4 show the profiles of the density, velocity, and pressure for the implementations of the current method, "GLF+WENO" and "GLF+adaptive WENO". The profiles of the theoretical solutions are also plotted for comparison. Note that "adaptive WENO" means that the $\epsilon$-adaptive technique (see Eq. (46)) was adopted. To exclude the possible numerical oscillations caused by FVS methods, GLF was used for all implementations. According to our analysis, numerical oscillations in the pressure and velocity would be expected if component-wise nonlinear WENO discretization is implemented. This is confirmed by Fig. 4, where distinct numerical oscillations can be observed near the location of the contact discontinuity $(x=0.5)$. In contrast, these nonphysical oscillations were successfully prevented when our consistent WENO scheme was used. In addition, compared with GLF+WENO, a remarkable reduction in the numerical oscillations of the velocity and pressure was observed when GLF+adaptive WENO was used. This agrees with Section 3.3.2. Therefore, Fig. 4 confirms that numerical oscillations were induced by component-wise nonlinear difference discretization and that the use of the consistent WENO scheme can successfully prevent this type of numerical oscillations.

Note that the $\epsilon$-adaptive technique adopted in this work is just a simplified version of that in [49]. This technique is preliminary so far and has only been subjected to a simple test of the advection of a square wave [49]. For complex problems, the switch between the optimal linear scheme (for smooth regions) and nonlinear WENO scheme may not work perfectly, and some oscillations may be induced around discontinuities. Thus, a systematic study is recommended (see [49] for more information) but is outside the scope of the present work.

\subsection{Material interface}

The second test was an advection problem where two gases (nitrogen and helium) were separated by a material interface $[31,42]$. The initial conditions were

$$
(\rho, u, p, \gamma)= \begin{cases}\left(7,1,1 / \gamma_{N_{2}}, 1.4\right)_{\mathrm{N}_{2}}, & -0.5 \leq x \leq 0.5 \\ \left(1,1,1 / \gamma_{N_{2}}, 1.66\right)_{\mathrm{He}}, & \text { otherwise. }\end{cases}
$$




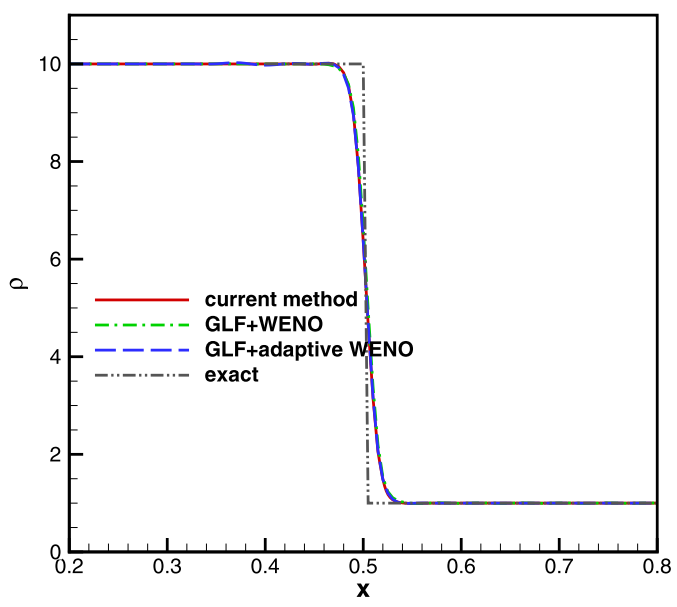

Fig. 3. Profiles of density at $t=0.4$ for implementations of current method, GLF+WENO, and GLF+adaptive WENO.
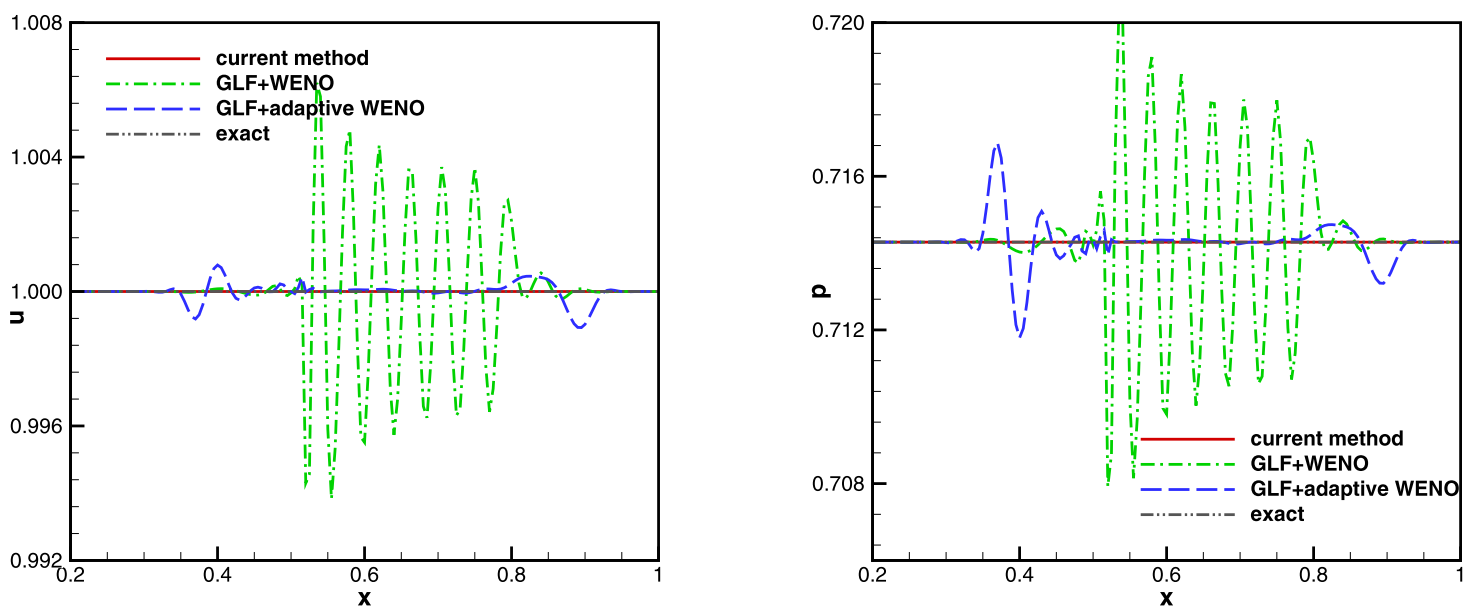

Fig. 4. Profiles of velocity (left) and pressure (right) at $t=0.4$ for implementations of current method, GLF+WENO, and GLF+adaptive WENO.

The computational domain was set to $-1 \leq x \leq 1$. The computation was performed on a uniform mesh with 101 grid points (grid spacing: $\Delta x=0.02$ ). Periodic boundary conditions were implemented on the left and right boundaries, and the final time was $t=2$.

Figs. 5 and 6 show the profiles of the density, velocity, and pressure as well as $\Gamma$ for the implementations of the current method and GLF+WENO. The profiles of the theoretical solutions are also plotted for comparison. Implementing the current method kept the pressure and velocity oscillation-free and thus validated the effectiveness of our proposed method for multi-material flows. In contrast, distinct numerical oscillations were observed in the component-wise implementation of the traditional WENO scheme. This highlights the importance of consistent implementation in compressible flows with discontinuities.

\subsection{Shock tube problem of two materials}

We used a two-material shock tube problem to demonstrate the capability of the current method in terms of capturing shock waves while maintaining the oscillation-free property at the contact discontinuity/material interface [3]. The initial conditions were

$$
(\rho, u, p, \gamma)= \begin{cases}(1,0,1,1.4), & 0 \leq x \leq 1 \\ (0.125,0,0.1,1.66), & 1<x \leq 2 .\end{cases}
$$

The computational domain was set to $0 \leq x \leq 2$. The computation was performed on a uniform mesh with 201 grid points (grid spacing: $\Delta x=0.01$ ). The initial values were fixed at the left and right boundaries, and the final time was $t=0.4$.

Figs. 7-9 show the profiles of the density, $\Gamma$, the velocity, and pressure for the implementations of the current method and GLF+WENO. We shall refer to the solution computed by combining GLF and the first-order scheme with $10^{4}$ grid points as the "reference" solution. Generally speaking, the implementations of the current method had essentially the same 

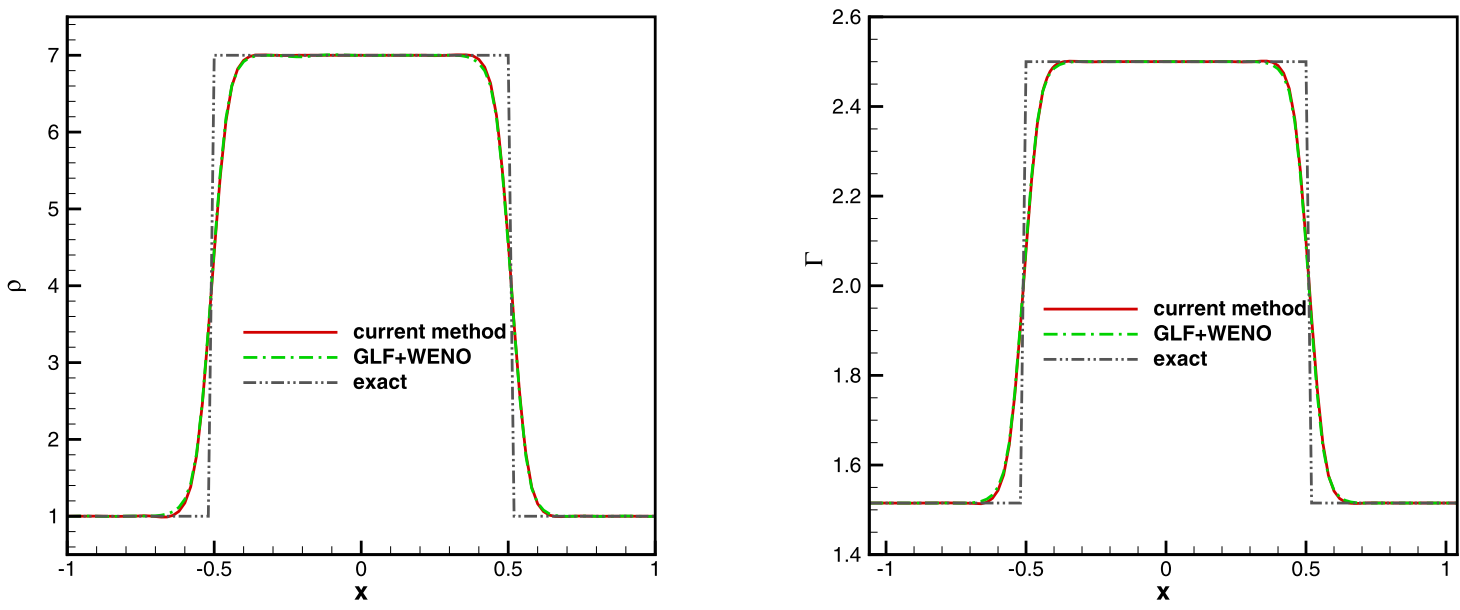

Fig. 5. Profiles of density (left) and $\Gamma$ (right) at $t=2$ for implementations of current method and GLF+WENO.
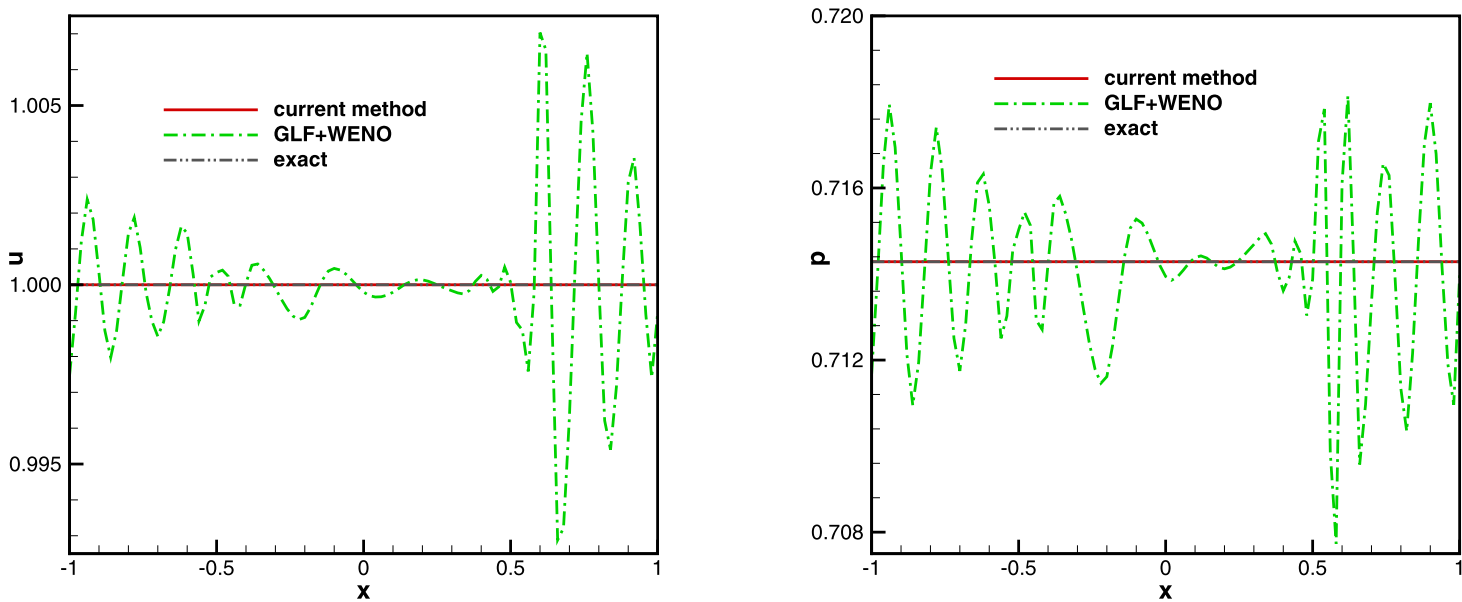

Fig. 6. Profiles of velocity (left) and pressure (right) at $t=2$ for implementations of current method and GLF+WENO.
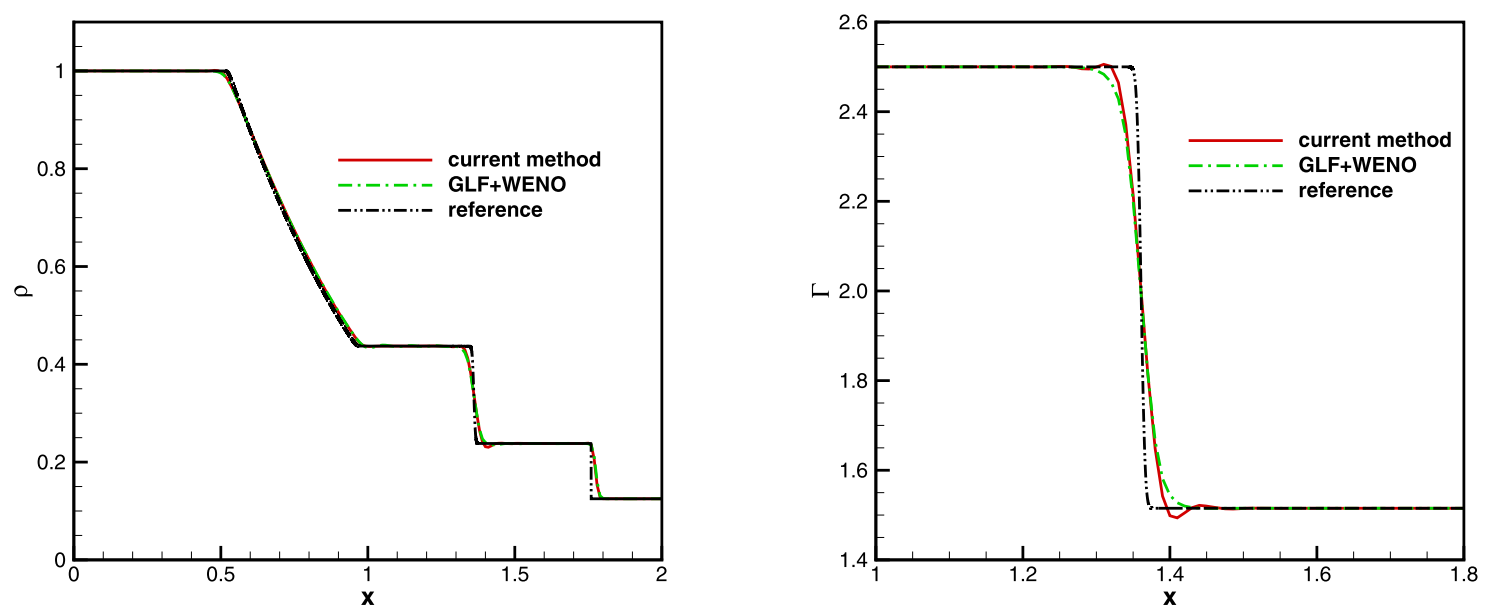

Fig. 7. Profiles of density (left) and $\Gamma$ (right) at $t=0.4$ for implementations of current method and GLF+WENO. 

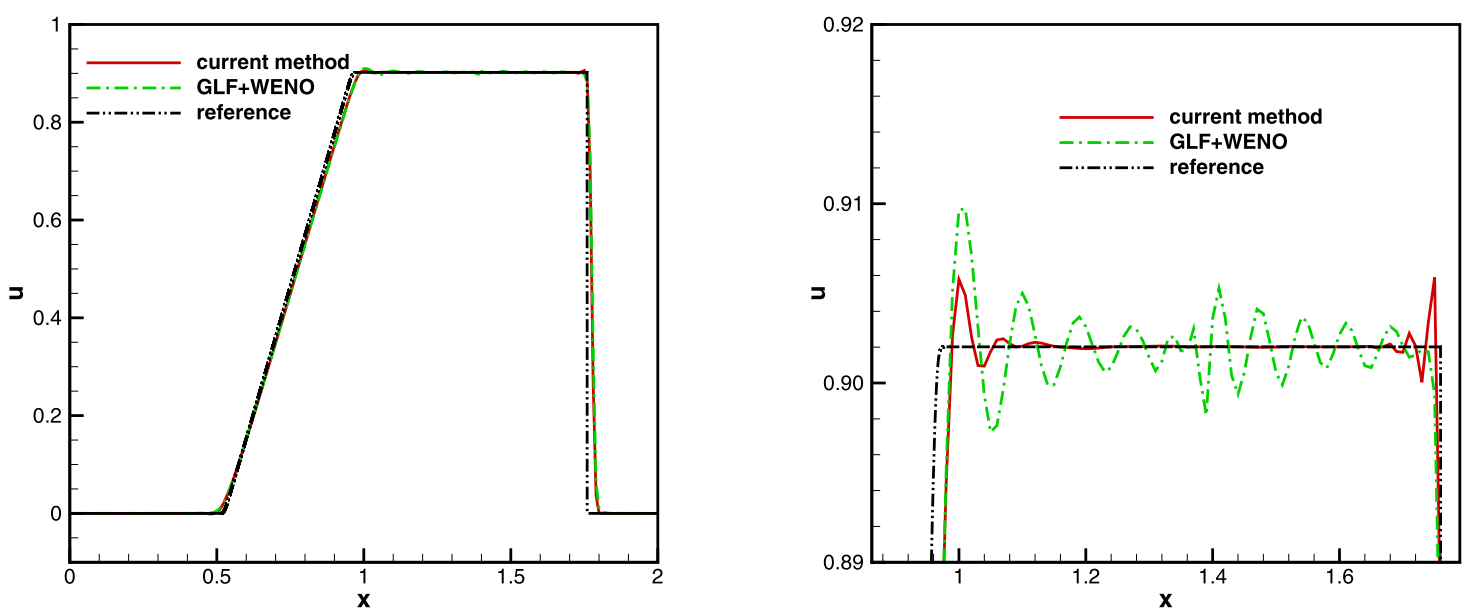

Fig. 8. Profiles of velocity at $t=0.4$ for implementations of current method and GLF+WENO (left) and close-up view around material interface (right).
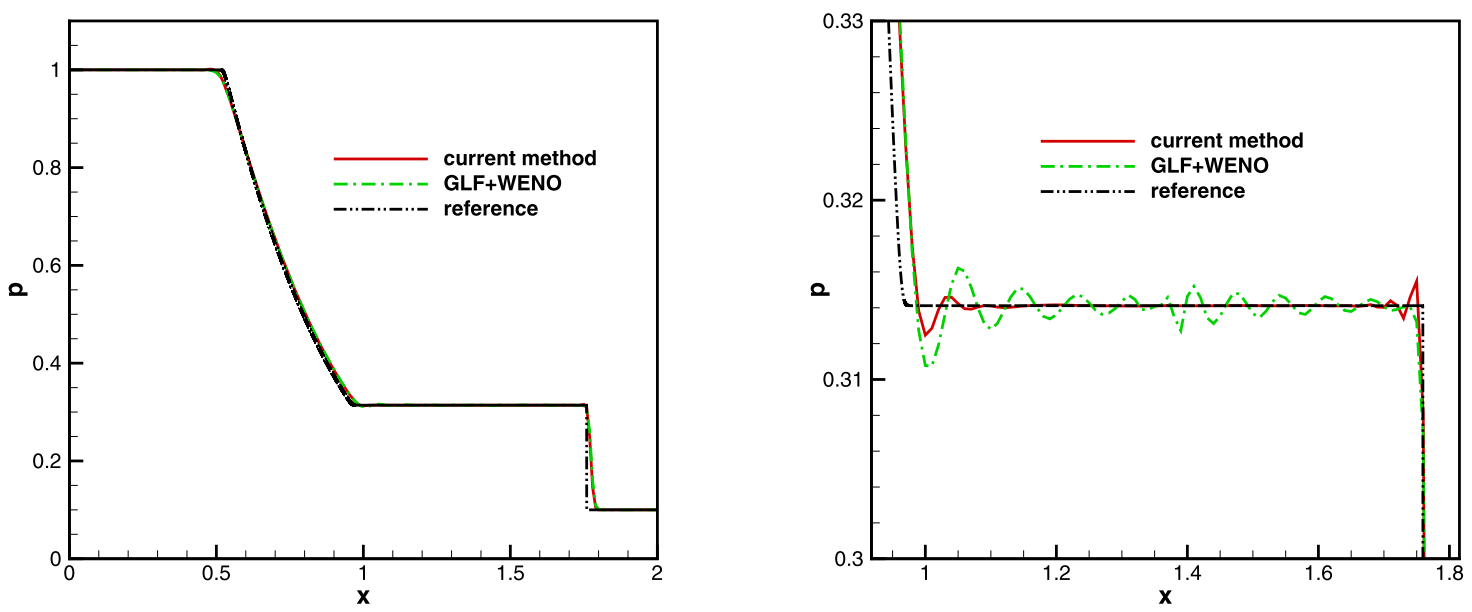

Fig. 9. Profiles of pressure at $t=0.4$ for implementations of current method and GLF+WENO (left) and close-up view around material interface (right).

accuracy as that of GLF+WENO over the whole computational domain. Thus, the effectiveness of the current method at capturing shock waves was validated. However, distinct numerical oscillations were observed when the profiles of the velocity and pressure obtained with GLF+WENO were examined, as shown in Figs. 8 and 9. Consequently, the use of the traditional component-wise WENO implementation is inappropriate for problems involving contact discontinuities or material interfaces, and we suggest adopting our proposed method. The current method does maintain oscillation-free pressure and velocity at the contact discontinuity.

\subsection{Two-dimensional Richtmyer-Meshkov instability}

The next case was the two-dimensional compressible multi-material Richtmyer-Meshkov instability problem [32]. The heavy and light fluids were $S F_{6}$ and air, respectively. A schematic of the initial flow configuration is shown in Fig. 10, and the initial shape of the perturbed interface was generated with

$$
y_{\text {dis }}=0.4+0.1 \sin (2 \pi(x+0.25)),-0.5<x<0.5 \text {. }
$$

The computational domain of this problem was $[-0.5,0.5] \times[0,16]$, and the initial conditions were

$$
(\rho, u, v, p, \gamma)= \begin{cases}\left(5.04,0,1.24,1 / \gamma_{\text {air }}, 1.093\right), & y<y_{\text {dis }} \\ \left(1,0,1.24,1 / \gamma_{\text {air }}, 1.4\right), & y_{\text {dis }}<y<0.7 \\ \left(1.4112,0,0.8787,1.6272 / \gamma_{\text {air }}, 1.4\right), & 0.7 \leq y \leq 16 .\end{cases}
$$

Periodic boundary conditions were imposed at $x=-0.5$ and $x=0.5$, while the initial values were fixed at $y=0$ and $y=16$.

In this problem, the development of instability was sensitive to the initial perturbation, which is completely determined by a sine function with a given wavelength and amplitude in this configuration. To produce the critical dynamics of such 


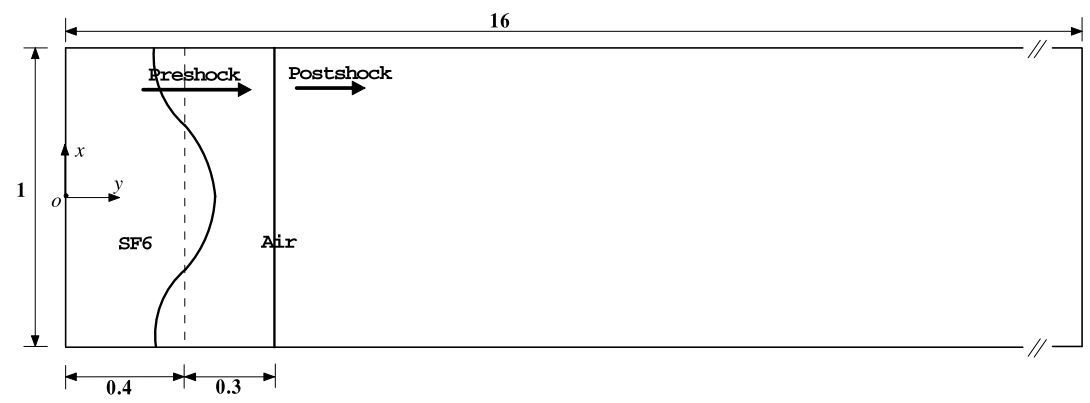

Fig. 10. Schematic of initial flow configuration for compressible multi-material Richtmyer-Meshkov instability.
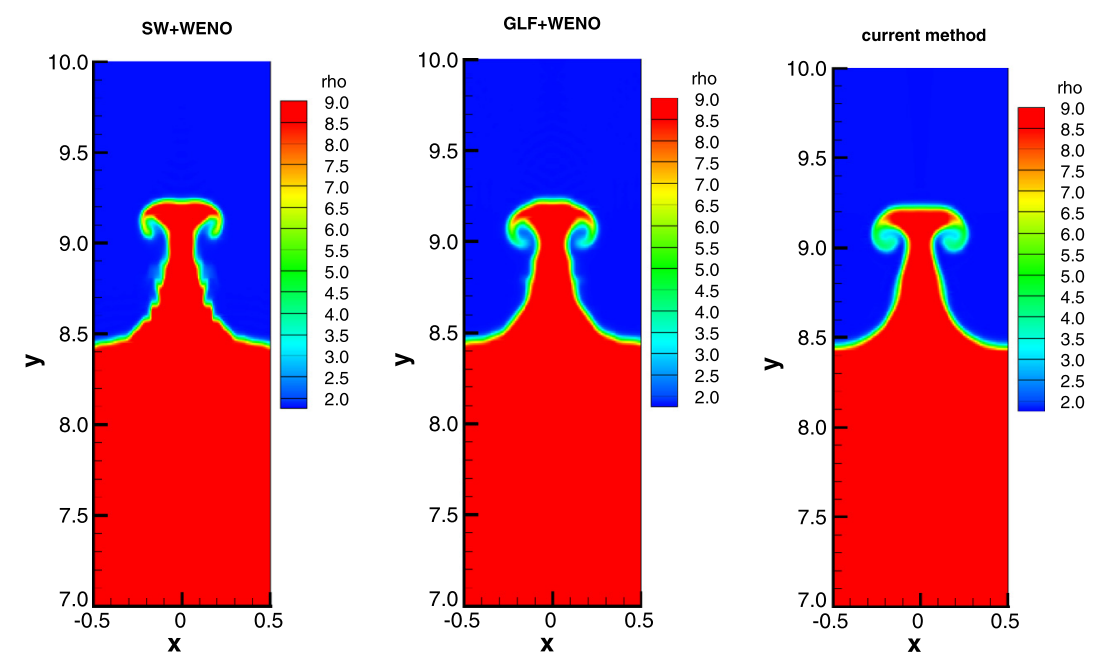

Fig. 11. Contours of density field at $t=8.25$ for implementations of SW+WENO (left), GLF+WENO (middle), and current method (right). Contours ranged from 1.5 to 8.5 .

a flow, any possible nonphysical numerical oscillations that may interact with the instability in a nonlinear manner and eventually contaminate the flow field should be avoided. In addition, if some numerical oscillations are induced, a full grid-independent solution cannot be achieved with grid refinement [57].

The test was first performed on a coarse mesh with uniform grid spacing $\left(\Delta x=\Delta y=\frac{1}{128}\right)$, and the results at $t=8.25$ are shown here and analyzed. Fig. 11 shows the contours of the density field for the implementations of SW+WENO, GLF+WENO, and the current method. We can observe small-scale structures in the results of SW+WENO and distinct small-scale structures in the results of GLF+WENO. In contrast, the flow transited smoothly in the results of the current method. One question that naturally arises from this is whether or not the small-scale features are physical in nature.

To answer the above question, the profiles of the density, the velocity, the pressure, and $\Gamma$ were examined along the straight line of $x=0$, as shown in Figs. 12 and 13. In the figures, only the results obtained with the current method and GLF+WENO are plotted for visual clarity; however, we emphasize that the following discussion and conclusions are also appropriate for SW+WENO, for which severe numerical oscillations were observed. Generally speaking, the figures show that the two implementations were comparable in terms of the global performance. However, when details near the material interface were examined carefully, remarkable numerical oscillations, no matter which profile, were observed in the results obtained with GLF+WENO but not in the results with the current method. The numerical oscillations must therefore be nonphysical and should be prevented by using the current method.

A similar phenomenon was also observed for the typical structure of the bubble. Fig. 14 plots the profiles of the velocity and pressure along the straight line of $x=-0.5$. Spurious numerical oscillations were still observed at the material interface for the implementation of GLF+WENO. All of these oscillations should be avoided by using the current method.

The above analysis was further examined with a refined mesh having uniform grid spacing $\left(\Delta x=\Delta y=\frac{1}{512}\right)$. The results at $t=8.25$ are presented in Fig. 15. Although small-scale structures were observed with both methods, the small-scale features in the results obtained with GLF+WENO were clearly nonphysical. Furthermore, when the velocity and pressure profiles along the straight line of $x=0$ were plotted for quantitative comparison, the same conclusions as discussed above were obtained again. These comparisons further validate the correctness of our analysis and the effectiveness of our proposed method. 

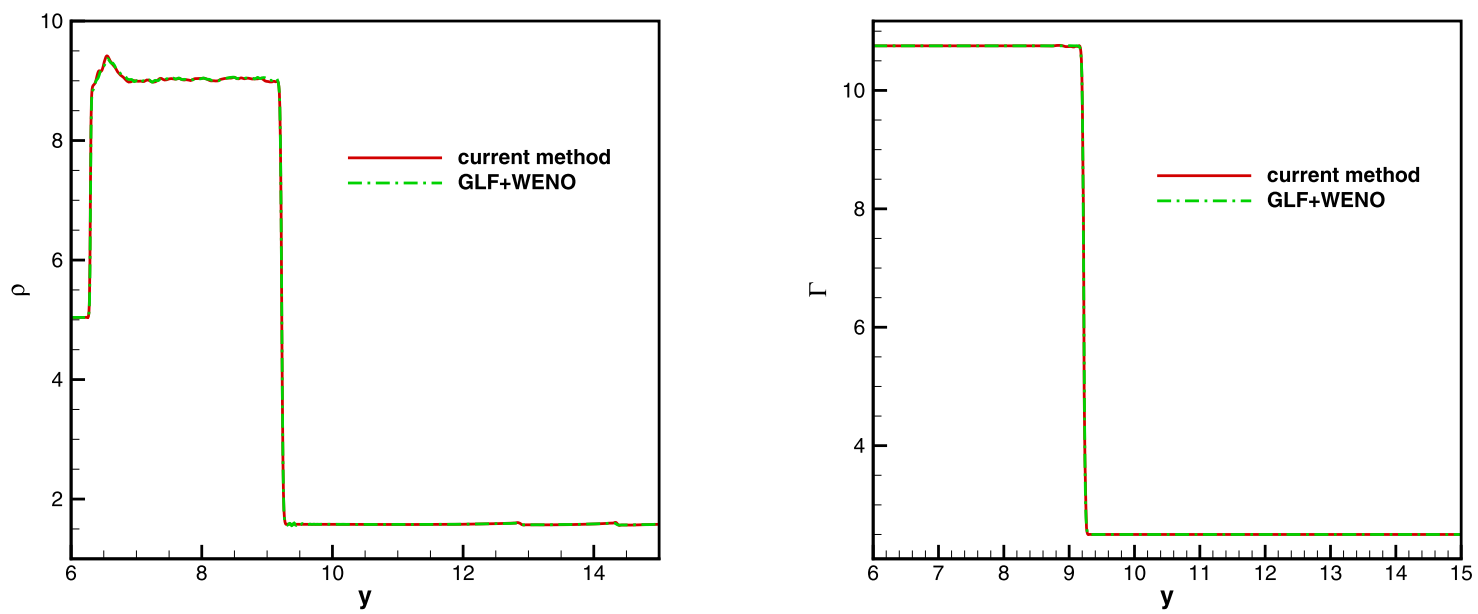

Fig. 12. Profiles of density (left) and $\Gamma$ (right) along the straight line of $x=0$ for implementations of current method and GLF+WENO.
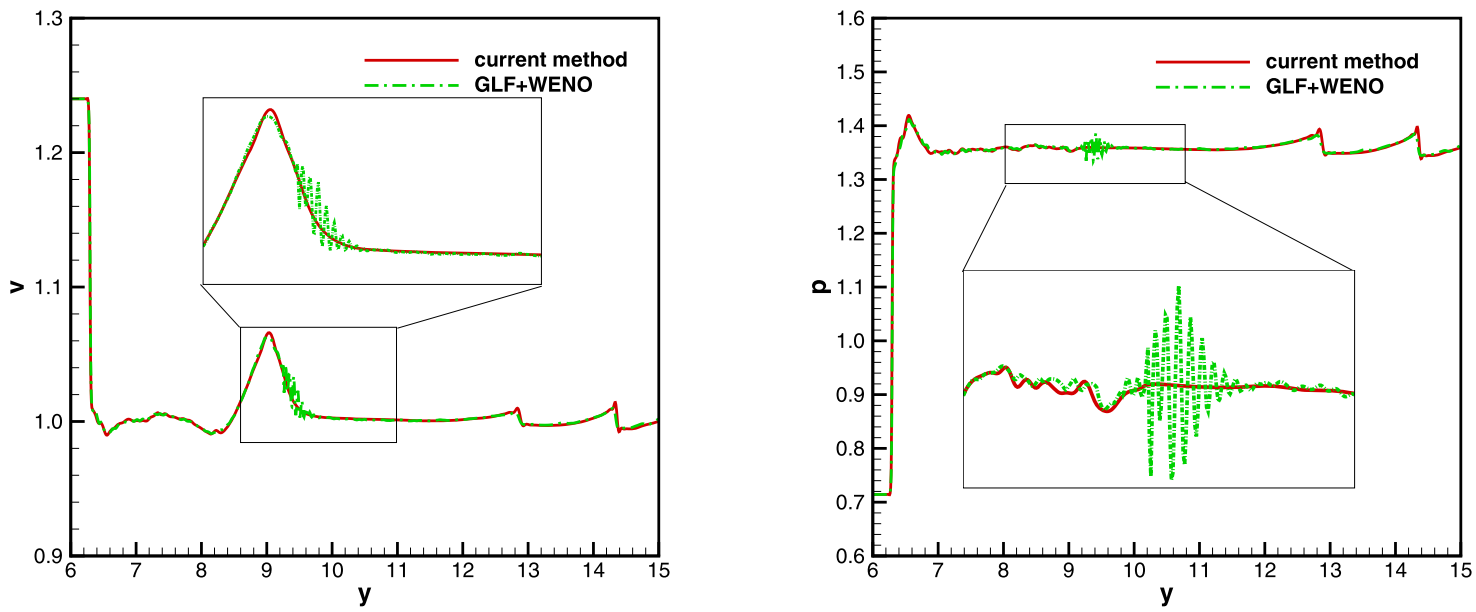

Fig. 13. Profiles of velocity (left) and pressure (right) along straight line of $x=0$ for implementations of current method and GLF+WENO.
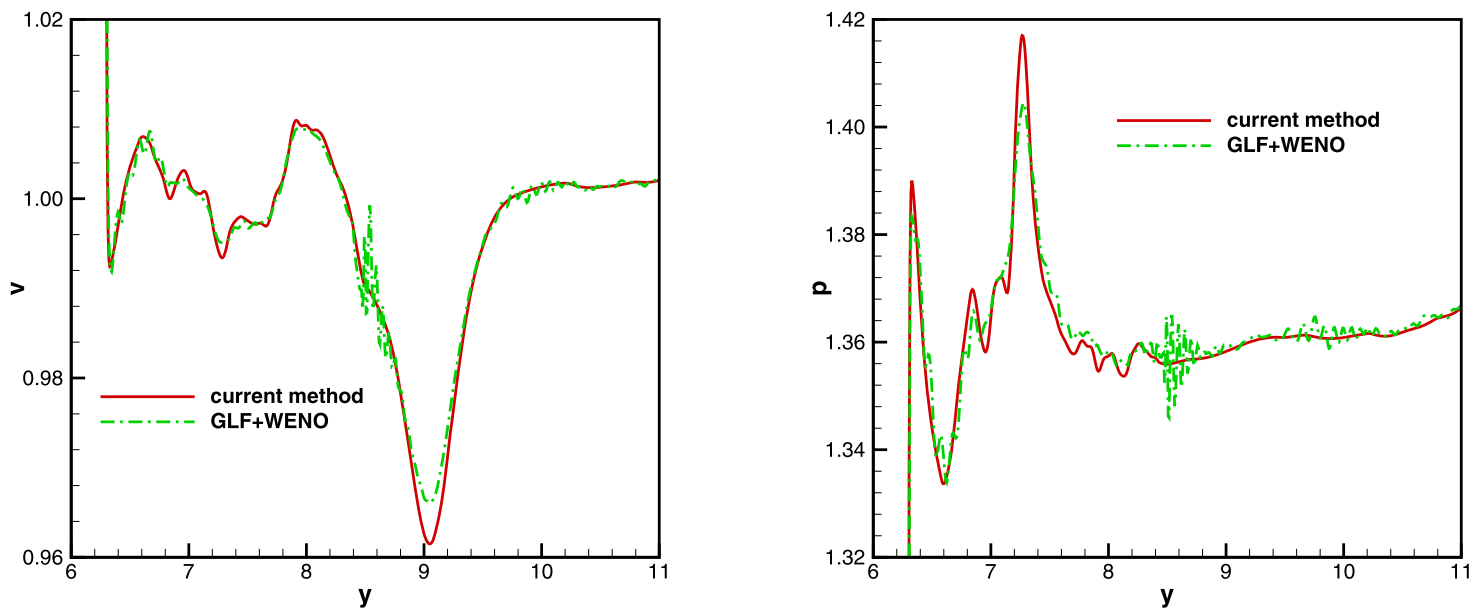

Fig. 14. Close-up view of velocity (left) and pressure (right) at $x=-0.5$ for implementations of current method and GLF+WENO. 

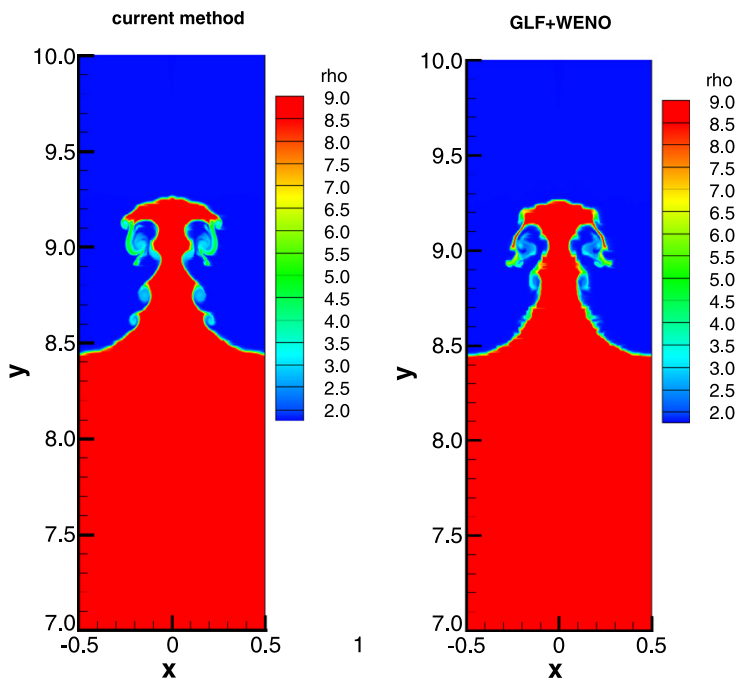

Fig. 15. Contours of density field at $t=8.25$ for implementations of current method (left) and GLF+WENO (right). Contours range from 1.5 to 8.5 .

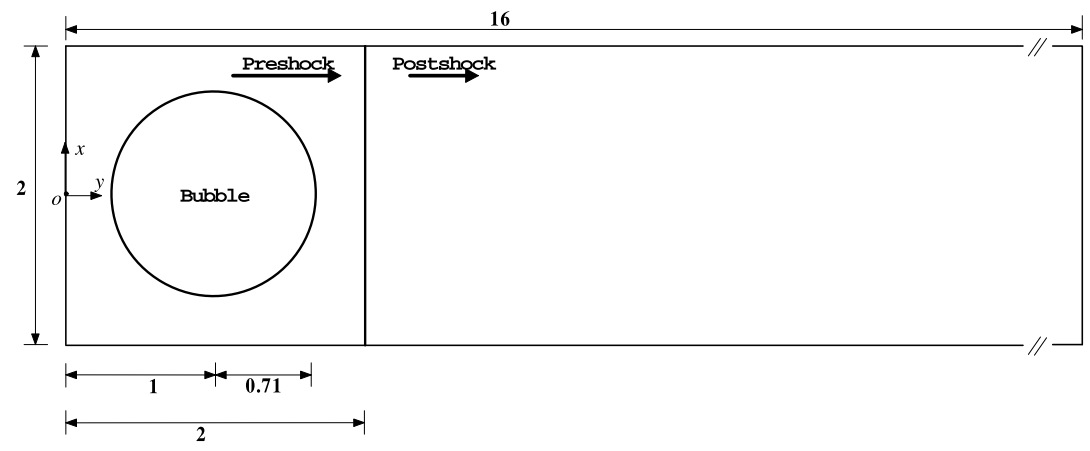

Fig. 16. Schematic of initial flow configuration for two-dimensional shock-bubble interaction problem.

\subsection{Shock-bubble interaction problem}

The final test was the two-dimensional shock-bubble interaction problem [32]. A schematic of the initial flow configuration is shown in Fig. 16. The initial conditions were

$$
(\rho, u, v, p, \gamma)= \begin{cases}\left(0.1819,0,1.22,1 / \gamma_{\text {air }}, 1.66\right), & (x-0)^{2}+(y-1)^{2}<0.5 \\ \left(1,0,1.22,1 / \gamma_{\text {air }}, 1.4\right), & y<2 \\ \left(1.3764,0,0.8864,1.5698 / \gamma_{\text {air }}, 1.4\right), & \text { otherwise. }\end{cases}
$$

The computational domain of this problem was $[-1,1] \times[0,16]$. Slip wall conditions were imposed at $x=-1$ and $x=1$, and the initial values were fixed at $y=0$ and $y=16$. The final time was $t=6.929$.

First, the numerical simulation of this problem was performed on an uniform mesh with grid points of $257 \times 2049$. Fig. 17 shows the contour plot of the density fields obtained with GLF+WENO and the current method. Obviously, nonphysical small-scale structures were qualitatively observed in the GLF+WENO result. The nonphysical numerical oscillations were further quantitatively confirmed by the pressure profiles, as shown in Fig. 18. These oscillations can be avoided by using the current method.

Finally, the numerical simulation of this problem was performed on an uniform mesh with grid points of $513 \times 4097$. Fig. 19 displays the contour plot of the density fields in the same region as that of Fig. 17. The same numerical oscillations were observed in the results of GLF+WENO and were successfully prevented by using the current method.

\section{Conclusions}

High-order nonlinear finite difference WENO schemes combined with point-wise FVS and component-wise discretization have been widely adopted in practical applications. However, we found that this traditional implementation leads to nonphysical numerical oscillations in compressible flows with contact discontinuities or material interfaces. In this paper, 

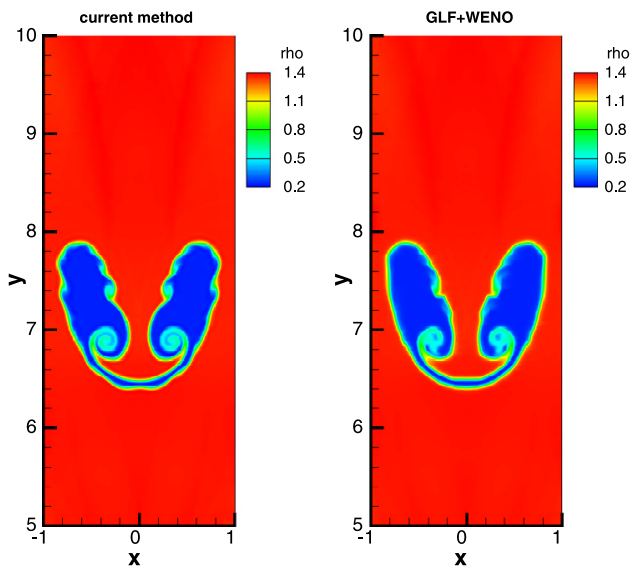

Fig. 17. Contours of density field at $t=6.929$ for implementations of current method (left) and GLF+WENO (right). Contours range from 0.2 to 1.4 .

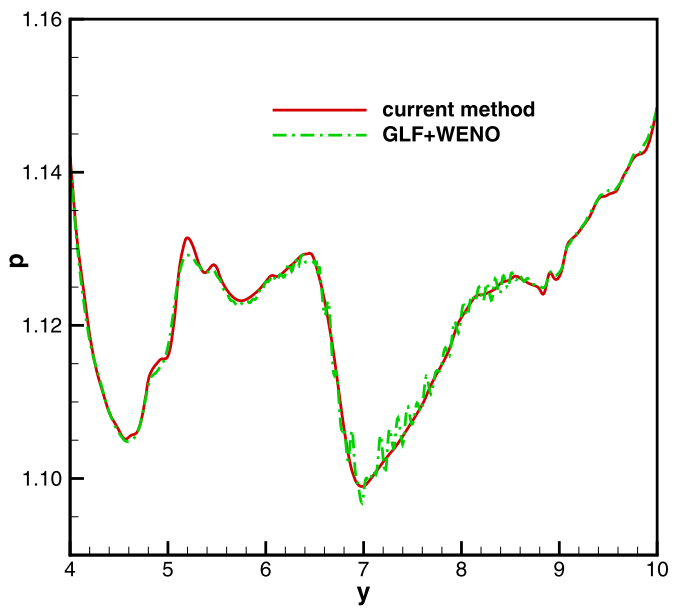

Fig. 18. Profiles of pressure along straight line of $x=0$ for implementations of current method and GLF+WENO.
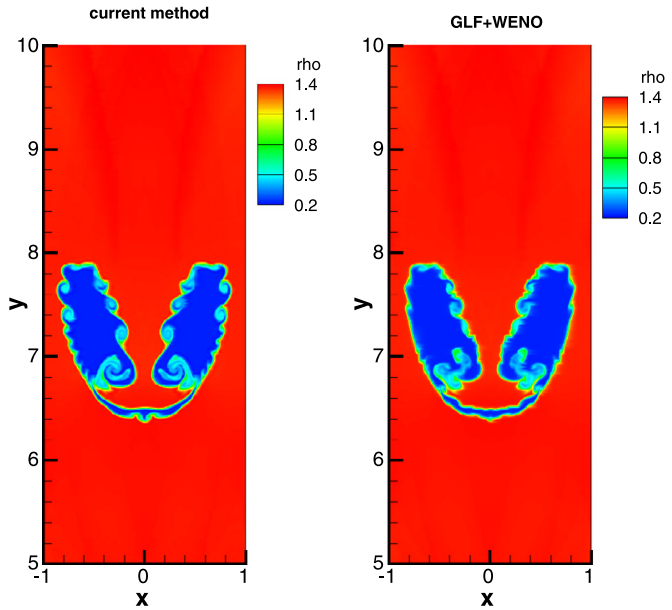

Fig. 19. Contours of density field at $t=6.929$ for implementations of current method (left) and GLF+WENO (right) with refined grids. The contours range from 0.2 to 1.4 
we reveal that two independent sources are responsible for the numerical oscillations and suggest two practical numerical strategies to prevent them.

The first numerical oscillations are essentially due to the incompatibility of a point-wise splitting of eigenvalues, which results from the intrinsic change in the speed of sound at discontinuities. In order to achieve compatibility, one possible approach is to replace point-wise FVS with a global FVS method such as GLF.

The second numerical oscillations are essentially due to the inconsistency of component-wise nonlinear difference discretization among the equations of mass, momentum, energy, and fluid composition for multi-material flows. To prevent these numerical oscillations, consistent discretization between different equations must be guaranteed. Our analysis showed that consistent discretization is automatically satisfied with linear difference discretization but not component-wise nonlinear difference discretization. To reconcile the contradiction between the consistency principle and necessity of the nonlinear difference schemes for capturing discontinuities, we propose a consistent implementation for nonlinear WENO schemes. In this implementation, only one set of common weights is used, in contrast to the sets of WENO weights matching the number of equations for component-wise WENO implementation.

In principle, all of the statements presented in this paper are appropriate for either single- (e.g., contact discontinuity) or multi-material (e.g., material interface) discontinuities. For the latter, however, the additional fluid composition equation should be split compatibly and discretized consistently with the mass, momentum, and energy equations. The results of various numerical tests including several contact discontinuities and multi-material flows confirmed the effectiveness, robustness, and low computation cost of our proposed method.

\section{Acknowledgements}

This work was supported by CAEP under Grant No. 2012A020210, NSFC under Grant Nos. 11171037, 91130021, 11472059, 11372330, 11472278 and Key Lab Foundation under Grant No. 9140C690104150C69.

\section{References}

[1] M. Brouillette, The Richtmyer-Meshkov instability, Annu. Rev. Fluid Mech. 34 (2002) 445-468

[2] D.H. Sharp, An overview of Rayleigh-Taylor instability, Physica D 12 (1984) 3-10, IN1-IN10, 11-18.

[3] R. Abgrall, S. Karni, Computations of compressible multifluids, J. Comput. Phys. 169 (2001) 594-623.

[4] E.F. Toro, Anomalies of conservative methods: analysis, numerical evidence and possible cures, Comput. Fluid Dyn. J. 11 (2002) 128-143.

[5] E. Turkel, Preconditioned methods for solving the incompressible and low speed compressible equations, J. Comput. Phys. 72 (1987) 277-298.

[6] J. Sesterhenn, B. Müller, H. Thomann, On the cancellation problem in calculating compressible low Mach number flows, J. Comput. Phys. 151 (1999) 597-615.

[7] F. Rieper, On the dissipation mechanism of upwind-schemes in the low Mach number regime: a comparison between Roe and HLL, J. Comput. Phys. 229 (2010) 221-232

[8] H. Guillard, C. Viozat, On the behaviour of upwind schemes in the low Mach number limit, Comput. Fluids 28 (1999) 63-86.

[9] B. Thornber, D. Drikakis, R. Williams, D. Youngs, On entropy generation and dissipation of kinetic energy in high-resolution shock-capturing schemes, J. Comput. Phys. 227 (2008) 4853-4872

[10] B. Thornber, A. Mosedale, D. Drikakis, D. Youngs, R. Williams, An improved reconstruction method for compressible flows with low Mach number features, J. Comput. Phys. 227 (2008) 4873-4894.

[11] H. Tang, On the sonic point glitch, J. Comput. Phys. 202 (2005) 507-532.

[12] V. Elling, The carbuncle phenomenon is incurable, Acta Math. Sci. 29 (2009) 1647-1656

[13] J.J. Quirk, A contribution to the great Riemann solver debate, Int. J. Numer. Methods Fluids 18 (1994) $555-574$

[14] K. Xu, J.-S. Hu, Projection dynamics in Godunov-type schemes, J. Comput. Phys. 142 (1998) 412-427.

[15] H. Tang, T. Liu, A note on the conservative schemes for the Euler equations, J. Comput. Phys. 218 (2006) $451-459$.

[16] D. Drikakis, M. Hahn, A. Mosedale, B. Thornber, Large eddy simulation using high resolution and high order methods, Philos. Trans. R. Soc. A 367 (2009) 2985-2997.

[17] S. Clerc, Accurate computation of contact discontinuities in flows with general equations of state, Comput. Methods Appl. Mech. Eng. 178 (1999) $245-255$.

[18] E. Johnsen, On the treatment of contact discontinuities using WENO schemes, J. Comput. Phys. 230 (2011) $8665-8668$.

[19] E. Johnsen, T. Colonius, Implementation of WENO schemes in compressible multicomponent flow problems, J. Comput. Phys. 219 (2006) 715-732.

[20] R. Abgrall, How to prevent pressure oscillations in multicomponent flow calculations: a quasi conservative approach, J. Comput. Phys. 125 (1996) $150-160$.

[21] S. Karni, Multi-component flow calculations by a consistent primitive algorithm, J. Comput. Phys. 112 (1994) $31-43$.

[22] E.F. Toro, Defects of conservative approaches and adaptive primitive-conservative schemes for computing solutions to hyperbolic conservation laws, Technical Report MMU 9401, Manchester Metropolitan University, UK, 1994.

[23] S. Karni, Hybrid multifluid algorithms, SIAM J. Sci. Comput. 17 (1996) 1019-1039.

[24] P. Jenny, B. Müller, H. Thomann, Correction of conservative Euler solvers for gas mixtures, J. Comput. Phys. 132 (1997) $91-107$.

[25] R.P. Fedkiw, T. Aslam, B. Merriman, S. Osher, A non-oscillatory Eulerian approach to interfaces in multimaterial flows (the ghost fluid method), J. Comput. Phys. 152 (1999) 457-492.

[26] K.M. Shyue, An efficient shock-capturing algorithm for compressible multicomponent problems, J. Comput. Phys. 142 (1998) 208-242.

[27] K.M. Shyue, A fluid-mixture type algorithm for compressible multicomponent flow with van der Waals equation of state, J. Comput. Phys. 156 (1999) 43-88.

[28] K.M. Shyue, A fluid-mixture type algorithm for compressible multicomponent flow with Mie-Grüneisen equation of state, J. Comput. Phys. 171 (2001) $678-707$

[29] R. Saurel, R. Abgrall, A simple method for compressible multifluid flows, SIAM J. Sci. Comput. 21 (1999) 1115-1145.

[30] E. Toro, M. Spruce, W. Speares, Restoration of the contact surface in the HLL-Riemann solver, Shock Waves 4 (1994) 25-34.

[31] E. Johnsen, F. Ham, Preventing numerical errors generated by interface-capturing schemes in compressible multi-material flows, J. Comput. Phys. 231 (2012) 5705-5717. 
[32] T. Nonomura, S. Morizawa, H. Terashima, S. Obayashi, K. Fujii, Numerical (error) issues on compressible multicomponent flows using a high-order differencing scheme: weighted compact nonlinear scheme, J. Comput. Phys. 231 (2012) 3181-3210.

[33] X.-G. Deng, H.-X. Zhang, Developing high-order weighted compact nonlinear schemes, J. Comput. Phys. 165 (2000) $22-44$.

[34] P.E. Dimotakis, Turbulent mixing, Annu. Rev. Fluid Mech. 37 (2005) 329-356.

[35] C.-W. Shu, Essentially non-oscillatory and weighted essentially non-oscillatory schemes for hyperbolic conservation laws, ICASE Report No. 97-65, 1997.

[36] S. Pirozzoli, Numerical methods for high-speed flows, Annu. Rev. Fluid Mech. 43 (2011) 163-194.

[37] S. Pirozzoli, F. Grasso, T.B. Gatski, Direct numerical simulation and analysis of a spatially evolving supersonic turbulent boundary layer at $M=2.25$, Phys. Fluids 16 (2004) 530-545.

[38] X.-L. Li, D.-X. Fu, Y.-W. Ma, X. Liang, Direct numerical simulation of compressible turbulent flows, Acta Mech. Sin. 26 (2010) 795-806.

[39] A. Marquina, P. Mulet, A flux-split algorithm applied to conservative models for multicomponent compressible flows, J. Comput. Phys. 185 (2003) $120-138$.

[40] R. Donat, A. Marquina, Capturing shock reflections: an improved flux formula, J. Comput. Phys. 125 (1996) $42-58$.

[41] G.-S. Jiang, C.-W. Shu, Efficient implementation of weighted ENO schemes, J. Comput. Phys. 126 (1996) $202-228$.

[42] H. Terashima, S. Kawai, M. Koshi, Consistent numerical diffusion terms for simulating compressible multicomponent flows, Comput. Fluids 88 (2013) 484-495.

[43] J.L. Steger, R.F. Warming, Flux vector splitting of the inviscid gasdynamic equations with applications to finite difference methods, J. Comput. Phys. 40 (1981) 263-293.

[44] C.-W. Shu, S. Osher, Efficient implementation of essentially non-oscillatory shock-capturing schemes, II, J. Comput. Phys. 83 (1989) 32-78.

[45] A. Harten, P.D. Lax, B. van Leer, On upstream difference and Godunov-type schemes for hyperbolic conservation laws, SIAM Rev. 25 (1983) 35-61.

[46] D. Drikakis, S. Tsangaris, On the solution of the compressible Navier-Stokes equations using improved flux vector splitting methods, Appl. Math. Model. 17 (1993) 282-297.

[47] E.F. Toro, Riemann Solvers and Numerical Methods for Fluid Dynamics: A Practical Introduction, third edition, Springer-Verlag, 2009.

[48] D.S. Balsara, C.-W. Shu, Monotonicity preserving weighted essentially nonoscillatory schemes with increasingly high order of accuracy, J. Comput. Phys, 160 (2000) 405-452.

[49] F.-L. Jia, Z. Gao, W.-S. Don, A spectral study on the dissipation and dispersion of the WENO schemes, J. Sci. Comput. 63 (2014) $49-77$.

[50] Borges, M. Carmona, M. Costa, W.-S. Don, An improved weighted essentially non-oscillatory scheme for hyperbolic conservation laws, J. Comput. Phys. 227 (2008) 3191-3211.

[51] M. Castro, B. Costa, W.-S. Don, High order weighted essentially non-oscillatory WENO-Z schemes for hyperbolic conservation laws, J. Comput. Phys. 230 (2011) 1766-1792.

[52] W.-S. Don, R. Borges, Accuracy of the weighted essentially non-ossillatory conservative finite difference schemes, J. Comput. Phys. 250 (2013) 347-372.

[53] J.-C. Huang, H. Lin, J.-Y. Yang, Implicit preconditioned WENO scheme for steady viscous flow computation, J. Comput. Phys. 228 (2009) 420-438.

[54] J.-S. Shuen, K.-H. Chen, Y. Choi, A coupled implicit method for chemical non-equilibrium flows at all speeds, J. Comput. Phys. 106 (1993) $306-318$.

[55] A.K. Henrick, T.D. Aslam, J.M. Powers, Mapped weighted essentially non-oscillatory schemes: achieving optimal order near critical points, J. Comput. Phys. 207 (2005) 542-567.

[56] S. Gottlieb, C.-W. Shu, Total variation diminishing Runge-Kutta schemes, Math. Comput. 67 (1998) $73-85$.

[57] P. Movahed, E. Johnsen, A solution-adaptive method for efficient compressible multifluid simulations, which application to the Richtmyer-Meshkov instability, J. Comput. Phys. 239 (2013) 166-186. 